This document is confidential and is proprietary to the American Chemical Society and its authors. Do not copy or disclose without written permission. If you have received this item in error, notify the sender and delete all copies.

\title{
Does Twisted n-Conjugation Framework Always Induce Efficient Intersystem Crossing? A Case Study with Benzo[b]- and [a]phenanthrene-fused BODIPY Derivatives
}

\begin{tabular}{|c|c|}
\hline Journal: & The Journal of Physical Chemistry \\
\hline Manuscript ID & Draft \\
\hline Manuscript Type: & Article \\
\hline $\begin{array}{r}\text { Date Submitted by the } \\
\text { Author: }\end{array}$ & $\mathrm{n} / \mathrm{a}$ \\
\hline Complete List of Authors: & $\begin{array}{l}\text { yan, yuxin; Dalian University of Technology, State Key Laboratory of } \\
\text { Fine Chemicals } \\
\text { Sukhanov, Andrei; Zavoisky Physical-Technical Institute, } \\
\text { Bousquet, Manon; Universite de Nantes, Laboratoire CEISAM - UMR } 6230 \\
\text { Guan, Qing Lin; Liaoning Normal University, College of Chemistry and } \\
\text { Chemical engineering, } \\
\text { Zhao, Jianzhang; Dalian University of Technology, State Key Laboratory } \\
\text { of Fine Chemicals } \\
\text { Voronkova, Violeta; Kazanskij fiziko-tehnicheskij institut imeni E K } \\
\text { Zavojskogo KazNC RAN, } \\
\text { Escudero, Daniel; Katholieke Universiteit Leuven Faculteit } \\
\text { Wetenschappen, Department of Chemistry } \\
\text { Barbon, Antonio; Universita degli Studi di Padova, Dipartimento di } \\
\text { Scienze Chimiche } \\
\text { Xing, Yong-Heng; College of Chemistry and Chemical Engineering, } \\
\text { Liaoning Normal University, } \\
\text { Gurzadyan, Gagik; Dalian University of Technology, Institute of Artificial } \\
\text { Photosynthesis } \\
\text { Jacquemin, Denis; Universite de Nantes, Laboratoire CEISAM - UMR } \\
6230\end{array}$ \\
\hline
\end{tabular}

\section{SCHOLARONE \\ Manuscripts}




\title{
Does Twisted $\pi$-Conjugation Framework
}

\author{
Always Induce Efficient Intersystem
}

\section{Crossing? A Case Study with Benzo[b]- and}

\section{[a]phenanthrene-fused BODIPY Derivatives}

Yuxin Yan, ${ }_{\ddagger}^{+}$Andrei A. Sukhanov, ${ }_{\ddagger}^{b}$ Manon H. E. Bousquet, ${ }^{c}+$ Qinglin Guan, ${ }^{d}$ Jianzhang Zhao, ${ }^{*, a}$ Violeta K. Voronkova, ${ }^{*, b}$ Daniel Escudero, ${ }^{*, e}$ Antonio Barbon, ${ }^{*, f}$ Yongheng Xing, ${ }^{* d}$ Gagik G. Gurzadyan, ${ }^{* g}$ and Denis Jacquemin $*, c$

${ }^{a}$ State Key Laboratory of Fine Chemicals, School of Chemical Engineering, Dalian University of Technology, 2 Ling-Gong Road, Dalian 116024, P. R. China. E-mail: zhaojzh@dlut.edu.cn (J.Z.)

${ }^{b}$ Zavoisky Physical-Technical Institute FRC Kazan Scientific Center of RAS, Sibirsky Tract 10/7, Kazan 420029, Russia.E-mail:vio@kfti.knc.ru (V.KV.)

${ }^{c}$ Laboratoire CEISAM, CNRS, Université de Nantes, Nantes, France E-mail:

Denis.Jacquemin@univ-nantes.fr (D.J.)

${ }^{d}$ College of Chemistry and Chemical Engineering, Liaoning Normal University, Dalian 116029, P. R. China. E-mail: xingyongheng2000@163.com (Y.H.X.)

${ }^{e}$ Department of Chemistry, KU Leuven, Celestijnenlaan 200F, B-3001 Leuven, Belgium Email: Daniel.escudero@univ-nantes.fr(D.E.)

${ }^{f}$ Dipartimento di Scienze Chimiche, Università degli Studi di Padova, Via Marzolo 1, 35131 Padova, Italy.E-mail: antonio.barbon@unipd.it (A. B.)

${ }^{g}$ Institute of Artificial Photosynthesis, State Key Laboratory of Fine Chemicals, Dalian 116024, P. R. China Email: gurzadyan@dlut.edu.cn (G.G.G)

\$ These authors contributed equally to this work. 


\begin{abstract}
The photophysical properties, especially the intersystem crossing (ISC) of two heavy atom-free BODIPY derivatives with twisted $\pi$-conjugated frameworks (benzo $[b]$-fused BODIPY, BDP-B; and [a]phenanthrene-fused BODIPY, BDP-P) are studied with steady state and time-resolved optical and electron paramagnetic resonance (TREPR) spectroscopic methods as well as with $\mathrm{ADC}(2)$ theoretical investigations. Both derivatives show intense absorption of red light ( $\varepsilon=9.8 \times 10^{4} \mathrm{M}^{-1} \mathrm{~cm}^{-1}$ at $640 \mathrm{~nm}$ for BDP-P). Interestingly, BDP-B is with planar $\pi$-conjugation framework, but it displays weak fluorescence $\left(\Phi_{\mathrm{F}}<0.1 \%\right)$, shortlived singlet excited state (fluorescence lifetime, $\left.\tau_{\mathrm{F}}=0.2 \mathrm{~ns}\right)$ but long-lived triplet state $\left(\tau_{\mathrm{T}}=\right.$ $132.3 \mu \mathrm{s})$. In contrast, the more twisted BDP-P shows stronger fluorescence $\left(\Phi_{\mathrm{F}}=70 \%\right)$, longer singlet excited state lifetime $\left(\tau_{\mathrm{F}}=6.4 \mathrm{~ns}\right)$, but shorter triplet state lifetime $\left(\tau_{\mathrm{T}}=18.9\right.$ $\mu \mathrm{s})$. In contrast to helicenes ( $\Phi_{\mathrm{T}}=$ ca. $90 \%$ ), the ISC of BDP-P and BDP-B is non-efficient $\left(\Phi_{\mathrm{T}}<23 \%\right)$. The electron spin selectivity of the ISC of the derivatives is different, manifested by the phase pattern of the TREPR spectra as AAEAEE and EEEAAA for BDP-B and BDP$\mathbf{P}$, respectively. The spatially confined $T_{1}$ state wave function of the twisted molecule doesn't reduce the $T_{1}$ state energy $(1.44-1.61 \mathrm{eV})$, although the chromophore shows red-shifted absorption as compared to the native BODIPY (ca. $1.60 \mathrm{eV}$ ). This work demonstrated the twisted $\pi$-conjugated framework does not necessarily induce efficient ISC and we found a dark singlet state for Bodipy, which is rare.
\end{abstract}

\title{
1. INTRODUCTION
}


Triplet photosensitizers (PSs) have attracted much attention in fundamental photochemistry studies, ${ }^{1-3}$ as well as in applications in photocatalysis, ${ }^{4-6}$ photodynamic therapy (PDT), ${ }^{7-10}$ photovoltaics, ${ }^{11,12}$ photon upconversion, ${ }^{13-17}$ etc. For these applications, it is crucial to design organic triplet PSs showing strong visible light absorption, efficient intersystem crossing (ISC) and long-lived triplet states. ${ }^{1,2}$ ISC is an electron spin-forbidden electronic transition, whose efficiency relies on the magnitude of the spin-orbit coupling (SOC) effects in organic compounds. ${ }^{18}$ The El Sayed's rule (n- $\pi^{*} \leftrightarrow \pi-\pi^{*}$ transitions) and the heavy atom effect (compounds containing Pt, Ir, Ru, Br, I atoms, etc) both allow enhancing the ISC. ${ }^{19-24}$ However, in the framework of designing triplet PSs, these two strategies suffer from some drawbacks. On the one hand, compounds relying on $\mathrm{n}-\pi^{*} \leftrightarrow \pi-\pi^{*}$ transition-enhanced ISC present a weak visible light absorption. ${ }^{18,24}$ On the other hand, compounds with the heavy atom-effect present dark toxicity for their use in biological studies (e.g. PDT) and high cost of synthesis. ${ }^{8,9}$ Moreover, the heavy atom effect is not always efficient to induce ISC, especially in bulky chromophores, ${ }^{25,26}$ and it may actually reduce the triplet state lifetime, because both the $\mathrm{S}_{1} \rightarrow$ $\mathrm{T}_{1}$ and $\mathrm{T}_{1} \rightarrow \mathrm{S}_{0}$ transition are simultaneously enhanced. ${ }^{27-29}$ A short $\mathrm{T}_{1}$ lifetime is detrimental for the applications of triplet PSs, as the efficiency of the subsequent photophysical processes, e.g., intermolecular electron transfer and energy transfers, increases along the triplet state lifetimes of the PSs. ${ }^{30}$ It has indeed been demonstrated that longer triplet state lifetimes are beneficial for PDT, ${ }^{31,32}$ photocatalysis, ${ }^{33,34}$ and photon upconversion. ${ }^{17,30,35}$ 
Some strategies have been developed to achieve ISC in organic chromophores without invoking of heavy atom effect, such as those based on exciton coupling effect, ${ }^{36,37}$ electron spin converter, ${ }^{28,38,39}$ spin-orbit charge transfer, ${ }^{40-43}$ singlet fission, ${ }^{44-46}$ energy matching of the $\mathrm{S}_{1} / \mathrm{T}_{\mathrm{n}}$ states, ${ }^{47-49}$ and electron spin interaction between a chromophore and a radical. ${ }^{50,51}$ However, the molecular structures based on these ISC mechanisms are usually synthetically challenging. Therefore, it is highly desirable to develop simple and general strategies to design heavy atom-free organic triplet PSs. Clearly, it remains a major challenge in photochemistry to design heavy atom-free triplet photosensitizers.

Helicene compounds are known to present efficient ISC with quantum yields reaching up to $90 \%{ }^{52,53}$ It was proposed that the twisted structure of helicenes enhances the mixing between the singlet and triplet states, subsequently improving the ISC efficiency. ${ }^{52}$ Recent theoretical studies have indeed demonstrated that the SOC is increased by the non-coplanarity in helicenes molecules. ${ }^{53}$ However, helicenes are not an ideal molecular platform for designing triplet PSs because of their weak absorption of visible light and the complexity of further derivatization. ${ }^{54}$ Perylene-3,4,9,10-bis(dicarboximide) (PBI), a well-known fluorophore, shows high fluorescence quantum yields but poor ISC. However, and very interestingly, PBI derivatives with twisted $\pi$-conjugated framework were reported to show efficient ISC (singlet oxygen quantum yield $\Phi_{\Delta}$ is up to $67 \%$ ). ${ }^{45,54,55}$ Likewise, sizeable ISC yields were also reported in expanded porphyrin derivatives with twisted structure. ${ }^{56}$ However, to the best of our knowledge, very few chromophores have been studied for this twisted $\pi$-conjugated framework-induced 
ISC. It therefore remains an open question whether a twisted molecule will always show more efficient ISC than the corresponding planar structures or not, and this work investigates this question.

Scheme 1. Reported Twisted BODIPY Derivatives Showing Efficient ISC: Benzo $[a]$ phenanthrene-Fused BODIPY (P-BDP), Benzo $[a]$ anthracene-Fused BODIPY (A-BDP), Naphtha[b]-Fused BODIPY (BDP-N) and Dihydronaphtho[a]-Fused BODIPY (BDP-D) $)^{57-59}$

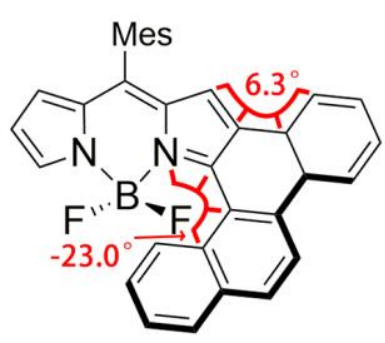

P-BDP $\Phi_{\Delta}=63 \%$

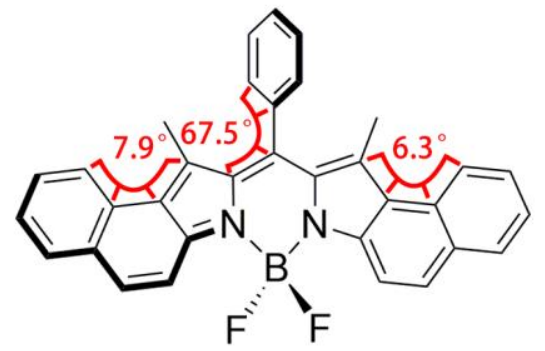

BDP-N $\quad \Phi_{\top}=52 \%$

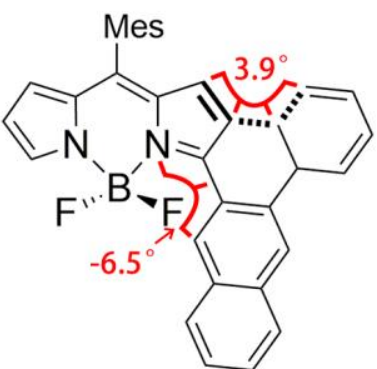

A-BDP $\quad \Phi_{\Delta}=23 \%$

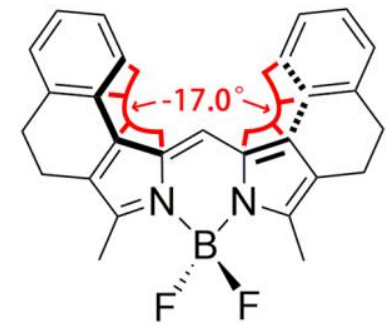

BDP-D $\quad \Phi_{\Delta}=55 \%$<smiles></smiles>

BODIPY

In this aspect, Boron dipyrromethene (BODIPY) dyes are of particular interest, as they show strong absorption of the visible light and readily derivatizable molecular structures. ${ }^{60-62}$ Several BODIPY-derived triplet PSs have been reported, based on the conventional heavy atom effect, ${ }^{63}$ exciton coupling, ${ }^{36,37}$ electron spin converter, ${ }^{64}$ as well as SOCT-ISC. ${ }^{40,41,43,65-67}$ 
BODIPY derivatives with twisted $\pi$-conjugated framework showing ISC ability are also known (Scheme 1). ${ }^{59,68,69}$ The more twisted BODIPY derivative, i.e., P-BDP, possesses the highest ISC efficiency among them (singlet oxygen quantum yield, $\Phi_{\Delta}=63 \%$ ). In comparison, the slightly twisted BODIPY (A-BDP) possesses a $\Phi_{\Delta}$ value of $23 \% .{ }^{59}$ Recently, some of us also reported ISC in other twisted BODIPY derivatives. For instance, a slightly twisted BODIPY derivative (BDP-N), shows an efficient ISC $\left(\Phi_{\mathrm{T}}=52 \%\right),{ }^{58}$ which is similar to the one of a BODIPY derivative (BDP-D) displaying a more distorted structure $\left(\Phi_{\Delta}=55 \%\right) .{ }^{57}$ Therefore, it is clear that additional examples should be studied to further elucidate the relationship between the degree of twisting and the ISC efficiency.

Herein, we selected two BODIPY derivatives, i.e., Benzo[b]-fused BODIPY (BDP-B) and [a]Phenanthrene-fused BODIPY (BDP-P, Scheme 2), both adopting a twisted geometry for the $\pi$-conjugated framework. Their photophysical properties, specially the ISC capability, are studied with various steady state, time-resolved optical/magnetic spectroscopies, ${ }^{68,69}$ as well as with theoretical approaches. Compared to the native BODIPY chromophore (Scheme 1), ${ }^{70-72}$ the $\pi$-conjugated system of these compounds is more extended, thus the absorption wavelength is red-shifted, a desired property for triplet PSs. Single crystal X-ray diffraction reveals that BDP-B is less twisted $\left(0.9^{\circ}\right.$ deviation from coplanarity $)$, whereas BDP-P is more twisted $\left(12.9^{\circ}\right.$ deviation from coplanarity) ${ }^{69}$ Interestingly, the two derivatives show different photophysical properties, for planar BDP-B, the fluorescence is very weak $\left(\Phi_{\mathrm{F}}<0.1 \%\right)$, and the singlet excited state is short-lived (fluorescence lifetime, $\tau_{\mathrm{F}}=0.2 \mathrm{~ns}$ ), but triplet state is long-lived $\left(\tau_{\mathrm{T}}\right.$ 
$=132.3 \mu \mathrm{s})$. On the contrary, the more twisted $\mathbf{B D P}-\mathbf{P}$ shows stronger fluorescence $\left(\Phi_{\mathrm{F}}=70 \%\right)$, longer singlet excited state lifetime ( $\left.\tau_{\mathrm{F}}=6.4 \mathrm{~ns}\right)$, but shorter triplet state lifetime $\left(\tau_{\mathrm{T}}=18.9 \mu \mathrm{s}\right)$. Meanwhile, a dark $\mathrm{S}_{1}$ state was identified for BDP-B, which is unusual for BDP chromophore, and only moderate ISC was observed for the two compounds $\left(\Phi_{\mathrm{T}}=23 \%\right.$ for BDP-B, $\Phi_{\Delta}<5.5 \%$ for BDP-P).

\section{METHODS}

2.1. Materials and Equipment. All of the chemicals used in synthesis are analytically pure and were used as received. The compounds BDP-B and BDP-P were prepared according to the previously reported methods. ${ }^{68,69,73}$ The molecular structure characterization data were supplied in the Supporting Information. The UV-Vis absorption spectra of compounds were recorded on a UV2550 spectrometer (Shimadzu Ltd., Japan). The fluorescence emission spectra were performed on a FS5 fluorescence spectrometer (Edinburgh Instruments Ltd., U.K.). The fluorescence lifetimes of the compounds were measured on an OB920 luminescence lifetime spectrometer (Edinburgh Instruments Ltd., U.K.). For other experimental procedures, refer to the Supporting Information.

2.2. Nanosecond Transient Absorption Spectroscopy. The nanosecond transient absorption spectra were measured on a LP980-K Laser Flash Photolysis Spectrometer (Kinetic mode, Edinburgh Instruments Ltd., UK). All samples were purged with $\mathrm{N}_{2}$ for 20 min for the collinear mode measurements, or $15 \mathrm{~min}$ in normal mode measurements before the measurements. The samples were excited with a nanosecond pulsed laser (OpoletteTM, the 
wavelength is tunable in the range of $210-2400 \mathrm{~nm}$. OPOTEK, USA). The typical laser power is ca. $5 \mathrm{~mJ}$ per pulse. The lifetimes (the decay traces of the transients were monitored) were obtained with the L900 software. The intrinsic triplet state lifetimes were obtained by fitting the decay traces with a special kinetics model. ${ }^{74}$

2.3. Femtosecond Transient Absorption Spectroscopy. A Ti-Sapphire amplified laser system with 100 fs pulse duration and $1 \mathrm{kHz}$ repetition rate (Spitfire Ace, Spectra-Physics) and a homemade pump-probe experimental setup, ${ }^{75}$ with a white light continuum probe (WLC), were used for the experiments. Wavelength of the pump beam was chosen according to the steady state absorption spectra of the studied compounds. The polarization direction between the probe and the pump beam was set as magic angle $\left(54.7^{\circ}\right)$ to avoid the effect of the molecular rotational diffusion. Experimental data were analyzed by using LabView software (National Instruments).

2.4. Single Crystal X-Ray Diffraction. A single crystal of BDP-B was obtained by slow diffusion between $n$-hexane and dichloromethane. The suitable single crystal of the compound BDP-B was determined and collected on a Bruker AXS SMART APEX II CCD diffractometer with graphite monochromatized Mo K $\alpha$ radiation $(\lambda=0.71073 \AA)$ at $298 \mathrm{~K}$. Semi-empirical absorption correction was applied using the program SADABS. The program SHELXTL-2018 was solved for space-group determination (XPREP), direct method structure solution (XS), and least-squares refinement (XL). ${ }^{76-78}$ CCDC 2036728 (BDP-B) contain the supplementary 
crystallographic data for this paper and the data can be obtained freely from the Cambridge Crystallographic Data Centre via https://www.ccdc.cam.ac.uk/.

2.5. Computational Details. Our post-Hartree-Fock calculations were performed using the TURBOMOLE package: ${ }^{79}$ the geometries of the ground and excited states were optimized at the MP2/cc-pVDZ and ADC(2)/cc-pVDZ levels, respectively, whereas the vertical transition energies were next determined at the $\mathrm{ADC}(2) / a u g$-cc-pVDZ level on these geometries. The lowest triplet geometry was also obtained at $\mathrm{ADC}(2)$ level (i.e., we did not use unrestricted MP2). All these calculations use the RI approximation, with the corresponding RI basis sets, using tight convergence criteria for the SCF procedure (so-called scfconv $=9$ ) and for the oneelectron density matrix (so-called denconv $=7$ ). Gaussian 16.A.03 was used to perform the DFT calculations of the spin densities. Finally, SOCs were computed with quadratic-response TD-DFT calculations ${ }^{80,81}$ (i.e., QR-TD-DFT) as implemented in the Dalton program. ${ }^{82}$ The latter calculations were performed at the $\mathrm{S}_{1}$ optimized geometries using the B3LYP functional in combination with the $6-31 \mathrm{G}^{*}(\mathrm{~d})$ atomic basis set.

2.6. TREPR Spectroscopy. For the TREPR spectra, BDP-B was dissolved in toluene/ methyltetrahydrofuran (TOL/MeTHF) mixed solvent $(1 / 2, \mathrm{v} / \mathrm{v})$ at $0.5 \mathrm{mM}$, BDP-P was dissolved in TOL/MeTHF $(1 / 2, \mathrm{v} / \mathrm{v})$ at $c=0.5 \mathrm{mM}$, with addition of $20 \%$ (by volume) iodoethane. The time-resolved continuous-wave (TR CW) EPR measurements were performed on a X-band EPR Elexsys E-580 spectrometer (Bruker) at $84 \mathrm{~K}$. The samples of BDP-B and BDP-P were photoexcited by pulsed laser at $570 \mathrm{~nm}$ and $590 \mathrm{~nm}$, respectively. The TREPR 
spectra of the triplet state were simulated using the EasySpin package based on Matlab (function pepper). ${ }^{83}$ The experiment structure field 'Exp.Ordering' of function pepper has been used to determine of TDM orientation in according to previously result. ${ }^{84}$ Fitting has been done using MATLAB's routine fmincon. Optical excitation was carried out with an optical parametric oscillator (OPO) system (LP603 SolarLS) pumped by an Nd:YAG laser (BriliantB Quantel) with a pulse energy of $1 \mathrm{~mJ}$. The repetition rate of the laser was set to $10 \mathrm{~Hz}$. Polarization for the magnetophotoselection experiments was obtained by using a Glan laser polarizer (SolarLS) in combination with a half-wave plate.

\section{RESULTS AND DISCUSSION}

3.1. Molecular Structure Designing Rationales. Native, or unsubstituted, BODIPY shows strong fluorescence negligible ISC capability (triplet state quantum yield $<2 \%$ ). ${ }^{60,62,85}$ Knowing that BODIPY derivatives BDP-B and BDP-P (Scheme 2), which present a twisted Scheme 2. Benzo[b]-Fused BODIPY Derivative (BDP-B) with Minor Twisted $\pi$ Conjugated Framework and Phenanthrene $[a]$-Fused BODIPY Derivative (BDP-P) with Significantly Twisted $\pi$-Conjugated Framework Used Herein. The Torsion Angles of the Dipyrrin Cores Determined by Single Crystal X-ray Diffraction are Presented ${ }^{69}$

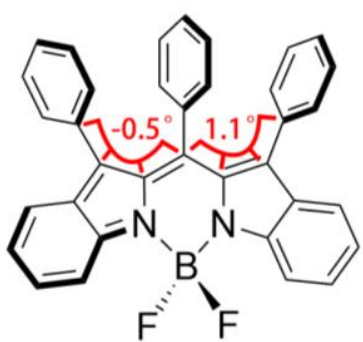

BDP-B

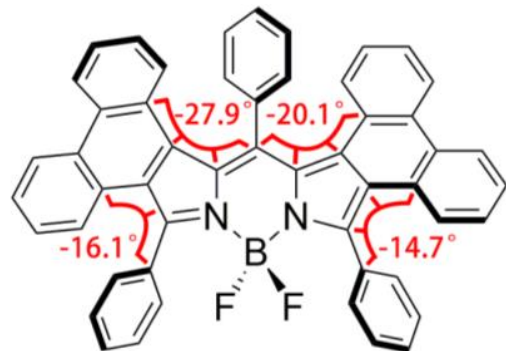

BDP-P 
$\pi$-conjugated framework, are weakly fluorescent (fluorescence quantum yields are $\sim 0 \%$ for BDP-B and 37\% for BDP-P, respectively), ${ }^{68,69}$ we envisage that ISC might be possible for these two compounds.

3.2. Molecular Structure of the Compounds. The molecular structure of BDP-P is more twisted: previous single crystal X-ray diffraction shows that the deviation of central $\mathrm{C}_{3} \mathrm{~N}_{2} \mathrm{~B}$ ring from the coplanarity is $12.9^{\circ},{ }^{69}$ whereas the deviation is much smaller in BDP-B (see below). The twisted structure of BDP-B was proposed by DFT calculations previously, but the degree of distortions of the molecular structure has not been discussed in detail. ${ }^{68} \mathrm{We}$ obtained a single crystal of the compound BDP-B, and the crystallographic data are listed in Table S1 whereas its molecular structure is displayed in Figure 1.

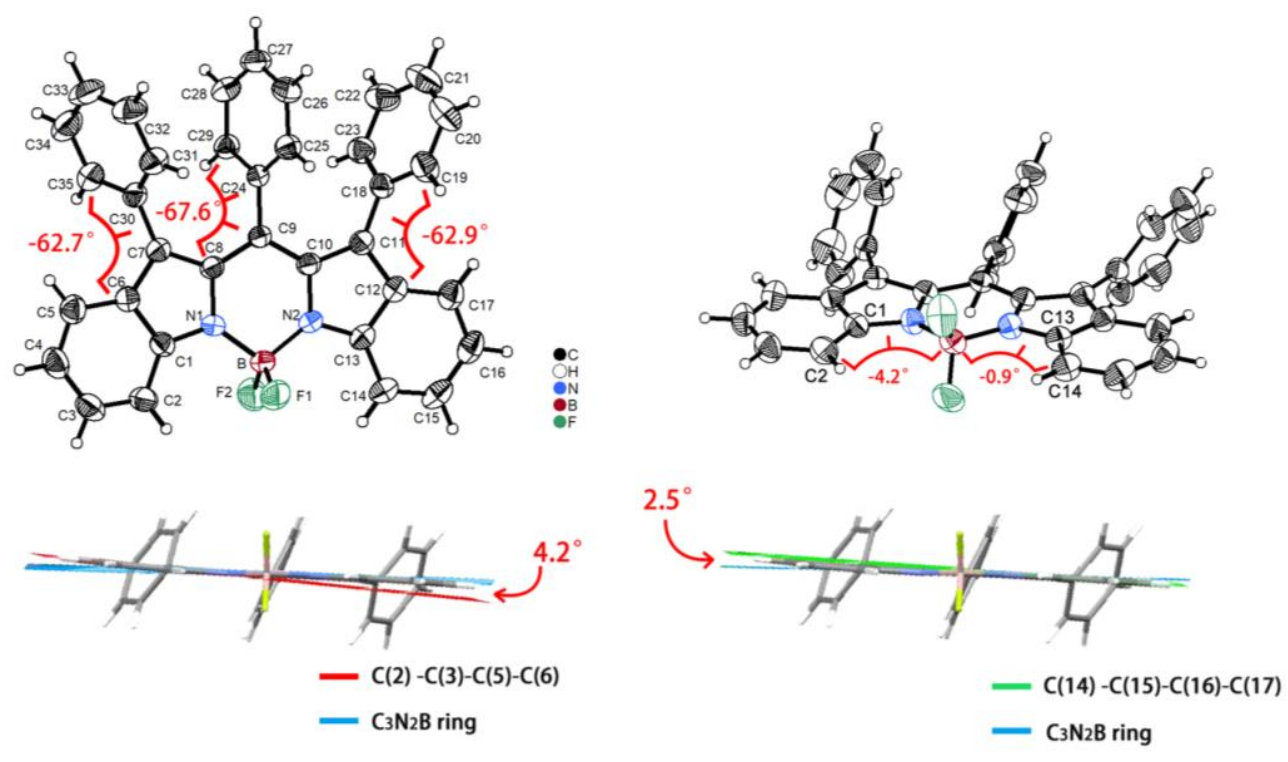

Figure 1. ORTEP view of the molecular structures of BDP-B determined by single-crystal Xray diffraction (50\% probability thermal ellipsoids). 
The single-crystal structure of BDP-B is slightly twisted (Figure 1). The benzene rings of $\mathrm{C}(14)-\mathrm{C}(15)-\mathrm{C}(16)-\mathrm{C}(17)$ and $\mathrm{C}(2)-\mathrm{C}(3)-\mathrm{C}(5)-\mathrm{C}(6)$ form dihedral angles of $2.5^{\circ}$ and $4.2^{\circ}$ with the plane of the central $\mathrm{C}_{3} \mathrm{~N}_{2} \mathrm{~B}$ ring, respectively. This torsion of the main $\pi$-conjugated framework is similar, yet smaller, than that in the recently reported BDP-N $\left(12.7^{\circ}\right.$ and $\left.2.0^{\circ}\right) .^{58,86}$ The central $\mathrm{C}_{3} \mathrm{~N}_{2} \mathrm{~B}$ ring is only twisted by $0.9^{\circ}$ out of coplanarity. The dihedral angle of the phenyl substituent at the meso- position and the main plane of the BODIPY molecule is $-67.6^{\circ}$, and the dihedral angles between the peripheral phenyl rings on both sides and the main BODIPY body are $-62.7^{\circ}$ and $-62.9^{\circ}$, respectively. The planes of the three phenyl rings are approximately parallel (Figure 1).

We also used second-order Møller-Plesset (MP2) to study the ground state geometry (Figure S16). Indeed, as the molecular geometry determined by the single crystal X-ray diffraction is affected by the molecular packing effect, it does not necessarily represent the molecular geometry in other media, and MP2 is known to be a very robust approach for ground-state geometries. The specific angle comparisons between the single crystal structure and the ground state geometry calculated by MP2 of BDP-B and BDP-P are listed in the Supporting Information (Figure S16). For BDP-B, the central $\mathrm{C}_{3} \mathrm{~N}_{2} \mathrm{~B}$ is more twisted in the optimized MP2 geometries than in the single crystal X-Ray diffraction studies, the $\mathrm{C}_{3} \mathrm{~N}_{2} \mathrm{~B}$ showing a $21.8^{\circ}$ deformation from planarity in the calculation. This is a rather typical trend as packing effects do favor more planar structures. For BDP-P, the MP2 optimized results are similar to those reported in literature, ${ }^{69}$ based on the single crystal structure analysis. 
3.3. UV-Vis Absorption and Fluorescence Emission Spectra. The UV-Vis absorption spectra of the compounds were studied (Figure 2). BDP-B gives an absorption band centered at $570 \mathrm{~nm}$ (the full width at half maximum, FWHM, is $2790 \mathrm{~cm}^{-1}$ ). ${ }^{68}$ Notably the absorption band is broad and weaker $\left(\varepsilon=3.8 \times 10^{4} \mathrm{M}^{-1} \mathrm{~cm}^{-1}\right)$ than the native BODIPY $\left(\varepsilon=7.9 \times 10^{4} \mathrm{M}^{-1}\right.$ $\mathrm{cm}^{-1}$. FWHM: $774 \mathrm{~cm}^{-1}$ ). The absorption band of the BDP-P is centered at $640 \mathrm{~nm}$ (molar absorption coefficient, $\varepsilon=9.8 \times 10^{4} \mathrm{M}^{-1} \mathrm{~cm}^{-1}$ ) twice as intense as in BDP-B (Table 1), but it is also much sharper (FWHM: $993 \mathrm{~cm}^{-1}$ ), which are typical feature for BODIPY dyes. Interestingly, the most deformed BDP-P has a more standard absorption spectrum than the least twisted BDP-B. As expected, both dyes are characterized by large red-shifts in both the absorption (65-144 nm) and emission (161-238 nm) as compared to the native BODIPY
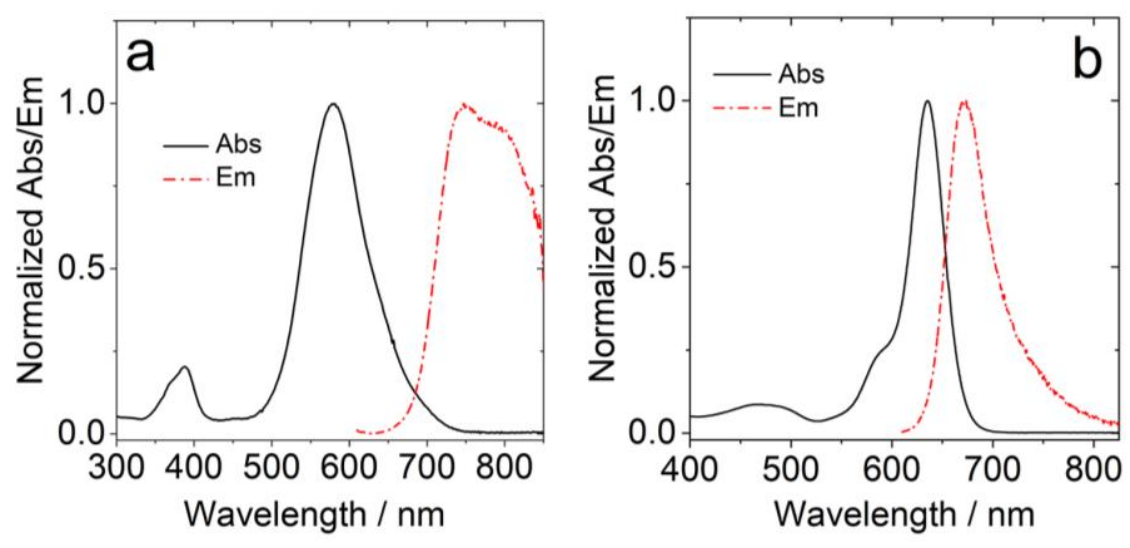

Figure 2. Normalized UV-Vis absorption spectra and fluorescence emission spectra of (a) BDP-B in toluene (TOL, $\lambda_{\mathrm{ex}}=600 \mathrm{~nm}$ ) and (b) BDP-P in acetonitrile (ACN, $\left.\lambda_{\mathrm{ex}}=600 \mathrm{~nm}\right), c$ $=1.0 \times 10^{-5} \mathrm{M}, 20^{\circ} \mathrm{C}$. 
(Figure S21), due to the extension of the $\pi$-conjugated systems. For BDP-B, the luminescence and the absorption bands are obviously not mirror-shape. Together with the large Stokes shift $\left(3884 \mathrm{~cm}^{-1}\right)$, a non-standard behavior for BODIPYs, this indicates a more significant geometry relaxation at the excited state, or that different excited states are responsible for the main absorption band and fluorescence emission. These postulates are rationalized below by the theoretical calculations.

Interestingly, the two compounds show drastically different fluorescence quantum yields. BDP-B is almost non-emissive $\left(\Phi_{\mathrm{F}}<0.1 \%\right.$, Table 1$) .{ }^{68}$ Previously it was reported that mesophenyl substituted BODIPYs show weak fluorescence due to the rotation of the phenyl moiety, or more precisely the torsion of the dipyrrin core at excited state, ${ }^{87,88}$ and that the fluorescence can be enhanced in viscous solvents. ${ }^{89}$ However, the fluorescence of BDP-B in a viscous solvent, polydimethylsiloxane (PDMS, 483cP), was also measured and the emission intensity does not increase significantly, indicating that the viscosity of solvents has no significant effect on the fluorescence of the compound (Supporting Information, Figure S17b). In other words, the fluorescence of BDP-B is not quenched by the rotation of the three phenyl rings in the molecule, or severe torsion of the $\mathrm{S}_{1}$ state geometry. ${ }^{87-89}$ The underlying reason of this specific behavior is actually a dark state quenching mechanism (see theory below) ${ }^{90}$ According to the quantum chemical-investigation of BDP-B, the absorption band (centered at $570 \mathrm{~nm}$ ) and the emission band (centered at $750 \mathrm{~nm}$ ) can be attributed to $\mathrm{S}_{0} \rightarrow \mathrm{S}_{3}$ and $\mathrm{S}_{1} \rightarrow \mathrm{S}_{0}$ transitions, respectively. Therefore, the stokes shift of BDP-B $\left(3884 \mathrm{~cm}^{-1}\right)$ is much larger than native 
BODIPY (467 $\mathrm{cm}^{-1}$, Figure S21). Interestingly, the fluorescence quantum yield of BDP-P is much higher $\left(\Phi_{\mathrm{F}}=64 \%\right.$, Table 1) ${ }^{69}$ although the molecular skeleton is severely distorted.

The fluorescence lifetime of both compounds was not reported. ${ }^{68,69}$ Therefore, we measured these fluorescence lifetimes using the time-correlated single photon counting (TCSPC) technique. The fluorescence lifetime of BDP-B is very short, the biexponential fitting gives 0.15 ns (population ratio: 98\%)/2.84 ns (2\%) (Instrument Response Function, IRF, 30 ps). This suggests that there is a fast decay channel for the emissive state of BDP-B. The lifetime of BDP-B at $77 \mathrm{~K}$ is slightly longer as compared to the lifetime at room temperature (0.91 ns vs $0.15 \mathrm{~ns}$ ) (Figure S19). The fluorescence lifetime of BDP-B is much shorter than the one of fluorescent BODIPY derivatives with co-planar molecular structures
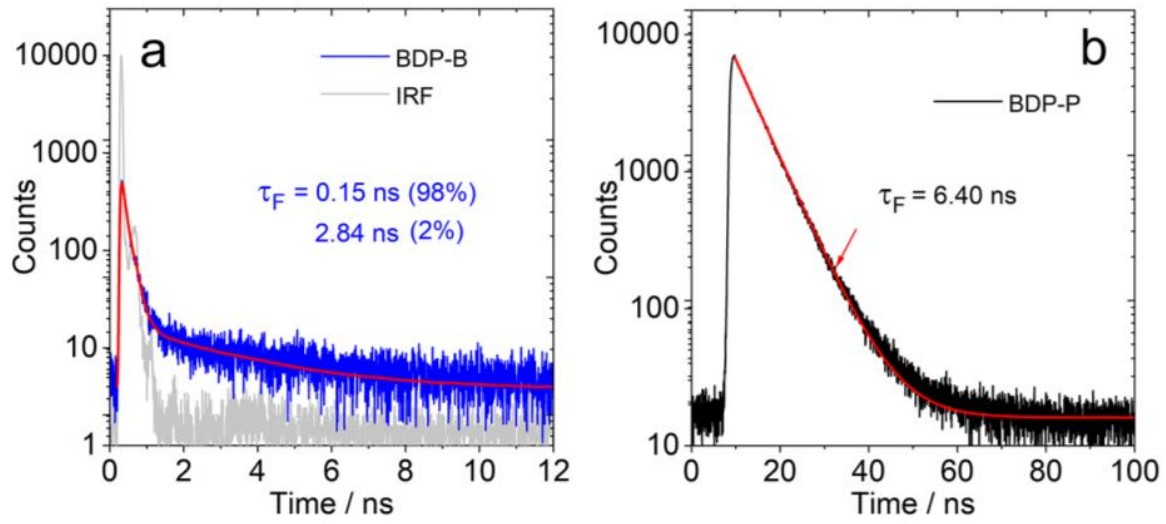

Figure 3. Decay traces of the fluorescence lifetime (a) BDP-B $\left(\lambda_{\mathrm{em}}=750 \mathrm{~nm}\right)$ in TOL and (b) BDP-P $\left(\lambda_{\mathrm{em}}=673 \mathrm{~nm}\right)$ in ACN. Excited with EPL picosecond pulsed lasers $(400 \mathrm{~nm}$ and 635 nm). $c=1.0 \times 10^{-5} \mathrm{M} .20^{\circ} \mathrm{C}$. 
(ca. $5 \mathrm{~ns}$ ), ${ }^{9,62,85}$ or the recently reported twisted BODIPY showing efficient ISC (ca. 3 ns). ${ }^{57,58}$

In contrast, the fluorescence of BDP-P decays with mono-exponential feature, the lifetime is longer (6.4 ns in acetonitrile, ACN), and shows no obvious solvent polarity dependence (Figure S18). Note the torsion of the molecular structure in BDP-P is more significant than BDP-B.

Table 1. Photophysical Data of the Benzo[b]- and Phenanthrene $[a]$-Fused BODIPY

\begin{tabular}{|c|c|c|c|c|c|c|c|c|c|}
\hline & Solvent $^{a}$ & $\lambda_{\mathrm{abs}}{ }^{b}$ & $\lambda_{\mathrm{em}}^{c}$ & $\varepsilon^{d}$ & $\Phi_{\Delta} \%$ & $\Phi_{\mathrm{T}} / \%$ & $\tau_{\mathrm{T}}{ }^{g} / \mu \mathrm{s}$ & $\Phi_{\mathrm{F}} / \%$ & $\tau_{\mathrm{F}} / \mathrm{ns}$ \\
\hline \multirow[t]{4}{*}{ BDP-B } & HEX & $569 / 384$ & 735 & $3.8 / 0.8$ & $2.6^{e}$ & $29.2^{f}$ & 108.5 & $0.10^{h}$ & $0.69^{i}$ \\
\hline & TOL & $579 / 388$ & 747 & $3.5 / 0.7$ & $5.8^{e}$ & $23.2^{f}$ & 132.3 & $0.09^{h}$ & $0.20^{j}$ \\
\hline & DCM & $575 / 386$ & 746 & $3.7 / 0.8$ & $2.8^{e}$ & $12.6^{f}$ & 89.3 & $0.02^{h}$ & $0.47^{i}$ \\
\hline & $\mathrm{ACN}$ & $562 / 384$ & 750 & $3.6 / 0.8$ & $2.3^{e}$ & $4.3^{f}$ & 84.7 & $--^{k}$ & $0.68^{i}$ \\
\hline \multirow[t]{4}{*}{ BDP-P } & HEX & 642 & 675 & 9.8 & $-^{k}$ & $--^{k}$ & 8.8 & $69.6^{h}$ & $6.69^{i}$ \\
\hline & TOL & 645 & 681 & 9.6 & $-{ }^{k}$ & $-^{k}$ & 12.9 & $74.0^{h}$ & $6.14^{i}$ \\
\hline & DCM & 641 & 680 & 9.1 & $-^{k}$ & $--^{k}$ & 11.0 & $72.3^{h}$ & $6.49^{i}$ \\
\hline & $\mathrm{ACN}$ & 635 & 670 & 8.7 & $5.5^{e}$ & $-^{k}$ & 8.7 & $64.4^{h}$ & $6.40^{i}$ \\
\hline
\end{tabular}

${ }^{a} E_{\mathrm{T}}(30)$ values of the solvents are $n$-HEX (31.0), TOL (33.9), DCM (40.7) and ACN (45.6), respectively, in kcal mol ${ }^{-1} .{ }^{b} c=1.0 \times 10^{-5} \mathrm{M}$, in $\mathrm{nm}$. ${ }^{c}$ Fluorescence emission wavelength, in nm. ${ }^{d}$ Molar absorption coefficient $\left(10^{4} \mathrm{M}^{-1} \mathrm{~cm}^{-1}\right)$. ${ }^{e}$ Singlet oxygen quantum yield. For BDP-B, Methylene Blue was used as standard ( $\Phi_{\Delta}=0.57$ in DCM). For BDP-P, 2,6-diiodo-bisstyryl BODIPY was used as standard $\left(\Phi_{\Delta}=0.624\right.$ in DCM). ${ }^{f}$ Triplet quantum yield determined with ground state bleaching, using nanosecond transient absorption spectra. Excitation at $551 \mathrm{~nm}$. 2,6-DiiodoBodipy was used as standard $\left(\Phi_{\mathrm{T}}=0.88\right.$ in TOL). ${ }^{g}$ Triplet excited state lifetimes. Measured by nanosecond transient absorption in deaerated solutions. ${ }^{h}$ Fluorescence quantum yields. For BDP-B, Methylene Blue was used as standard $\left(\Phi_{\mathrm{F}}=0.03\right.$ in Methanol). For BDP$\mathbf{P}$, absolute fluorescence quantum yields were observed. ${ }^{i}$ Fluorescence lifetimes (Instrument Response Function, IRF, 100 ps). ${ }^{j}$ Fluorescence lifetimes (Instrument Response Function, IRF, $30 \mathrm{ps}) .{ }^{k}$ Not observed. 
The photophysical properties of the compounds are summarized in Table 1. As a preliminary evaluation of the ISC ability, the photosensitized singlet oxygen $\left({ }^{1} \mathrm{O}_{2}\right)$ formation quantum yield $\left(\Phi_{\Delta}\right)$ was studied, using with 1,3-diphenylbenzoisofuran (DPBF) as an ${ }^{1} \mathrm{O}_{2}$ scavenger. $\Phi_{\Delta}$ of $5.8 \%$ for BDP-B (in TOL), and $\Phi_{\Delta}=5.5 \%$ for BDP-P were observed (in ACN), respectively. The $\mathrm{T}_{1}$ state energy of the two compounds was estimated with the ADC(2) method, as $1.03 \mathrm{eV}$ and $1.45 \mathrm{eV}$ for BDP-B and BDP-P at their $\mathrm{T}_{1}$ geometries, respectively (vide infra), in comparison the energy of ${ }^{1} \mathrm{O}_{2}$ is $0.98 \mathrm{eV} .{ }^{91}$ The rather low $\Phi_{\Delta}$ of BDP-B may therefore be due to the low $\mathrm{T}_{1}$ state energy rather than a poor ISC. Consequently, the triplet quantum yields were determined with the ground state depletion method using nanosecond transient absorption spectra. ${ }^{92}$ The $\Phi_{\mathrm{T}}$ of BDP-B is $23.2 \%$ in toluene (see Table 1). Negligible $\Phi_{\Delta}$ was observed for

\section{BDP-P.}

3.4. Femtosecond Transient Absorption Spectroscopy. In order to investigate the excited state kinetics and especially the ISC processes, femtosecond transient absorption spectroscopy was performed for both dyes (Figure 4). The excitation wavelength was selected at $650 \mathrm{~nm}$. In order to extract the kinetic information on the excited states evolution, the transient absorption data were analyzed with global fitting. ${ }^{93}$

For BDP-B (Figure 4a, in toluene), a ground state bleaching (GSB) band centered at $560 \mathrm{~nm}$ was observed upon photoexcitation, as well as a positive excited state absorption (ESA) band centered at $460 \mathrm{~nm}$, which is attributed to $S_{1} \rightarrow S_{3}$ absorption (see computation section). These two bands do not decay to the baseline within the available time window, indicating the 
existence of long-lived species. The evolution associated spectra (EAS) obtained for BDP-B are shown in Figure 4b. The first spectrum decays within 16.1 ps. The ESA band of the second spectrum is centered at $459 \mathrm{~nm}$, blue shifted by $6 \mathrm{~nm}$ as compared to the first spectrum. Thus, we propose that the vibrational relaxation and the internal conversion (IC) from $\mathrm{S}_{3}$ to $\mathrm{S}_{1}$ state takes place in ca. $16.1 \mathrm{ps}^{88}$ The second spectrum with a lifetime of $180.4 \mathrm{ps}$ is assigned to the
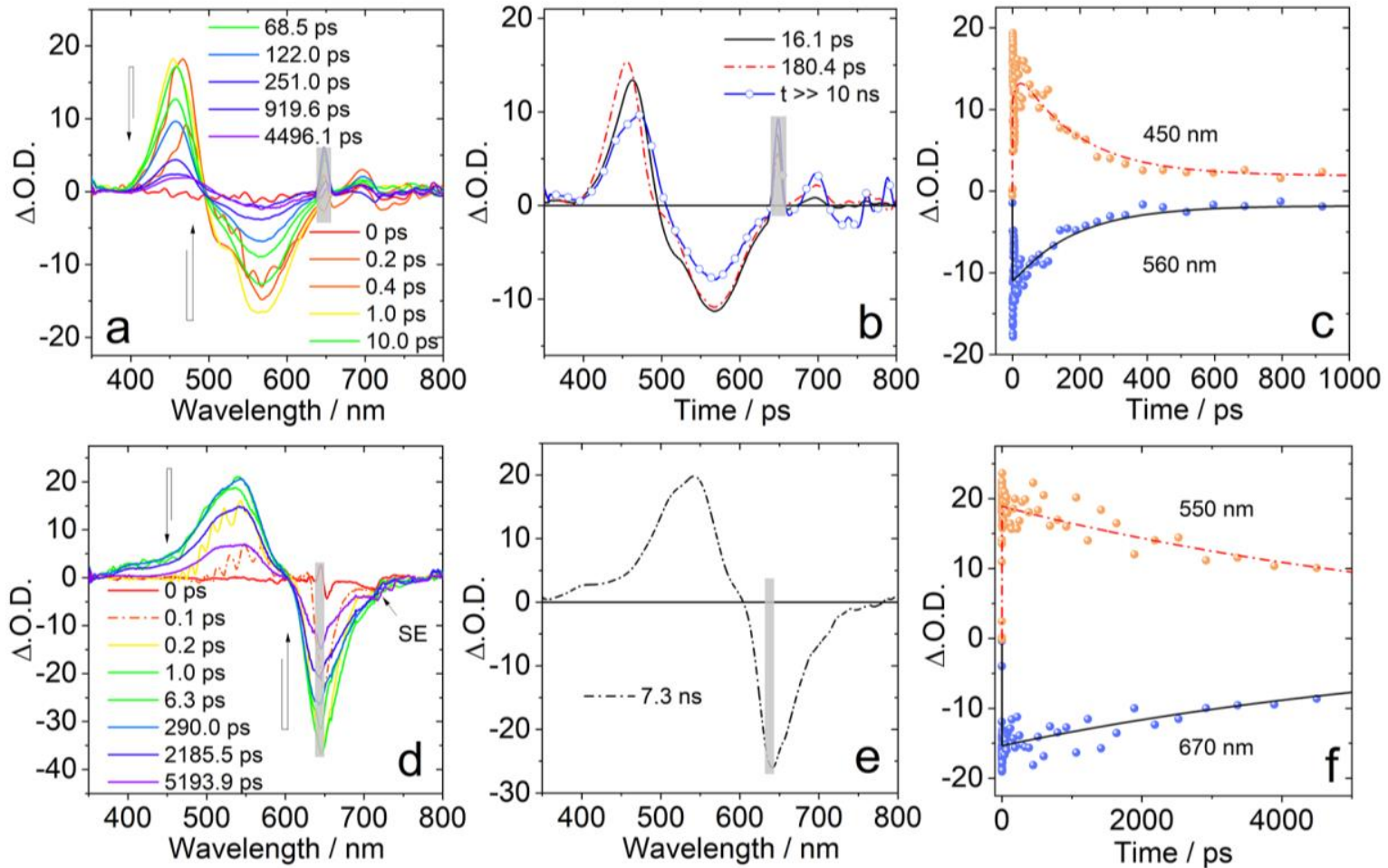

Figure 4. Femtosecond transient absorption spectra of (a) BDP-B in TOL and (d) BDP-P in ACN at different delay times. Evolution associated spectra (EAS) of (b) BDP-B in TOL and (e) BDP-P in ACN obtained from global analysis. Kinetic traces of (c) BDP-B in TOL and (f) BDP-P in ACN at selected wavelengths with fitting lines. $\lambda_{\text {ex }}=650 \mathrm{~nm}, 20{ }^{\circ} \mathrm{C}$. The scattered laser is at $650 \mathrm{~nm}$ (grey line). 
$\mathrm{S}_{1}$ state. Two competitive processes of $\mathrm{S}_{1} \rightarrow \mathrm{T}_{1}$ and $\mathrm{S}_{1} \rightarrow \mathrm{S}_{0}$ exist. The third species possesses a long lifetime, which is beyond the detection time window of the spectrometer, thus it is assigned to the triplet state $\left(\mathrm{T}_{1}\right)$ of BDP-B. This result is consistent with the nanosecond transient absorption spectra (Figure 5a). Hence, the ISC process from the singlet state to the triplet state takes place within 180.4 ps. This is close to the fast-decaying component of the fluorescence lifetime of BDP-B (150 ps). The ISC of BDP-B is faster than that of 2,6-diiodo8-thioBODIPY (500 ps) ${ }^{94}$ but it is slightly slower than that of 2,6-diiodoBODIPY (127 ps). ${ }^{95}$

For BDP-P (Figure 4d, in ACN), the GSB band is centered at $650 \mathrm{~nm}$ and a positive ESA band in the range of 360-600 nm was observed. The stimulated emission (SE) signal is in the 700-730 nm range. The EAS obtained for BDP-P in ACN are presented in Figure 5e. Only one species was observed. The lifetime is determined as $7.3 \mathrm{~ns}$, which is in good agreement with the fluorescence lifetime of $6.4 \mathrm{~ns}$ (Figure 4). Thus, the transient species is attributed to the $\mathrm{S}_{1}$ state of BDP-P, indicating that there is no generation of triplet state. The ultrafast spectra were also measured in ACN with 15\% iodoethane (v/v) for BDP-P (Supporting Information, Figure S24). Only one species is observed in the EAS indicating that the external heavy atom effect-induced ISC rate constant is slow and that it does not affect in picosecond scale, although triplet state was observed with nanosecond transient absorption spectra due to the external heavy atom effect.

\subsection{Nanosecond Transient Absorption Spectroscopy: the Triplet Excited State of the}

Compounds. We studied the triplet state of the compounds by using nanosecond transient 
absorption (ns TA) spectroscopy (Figure 5). For BDP-B, an ESA band in the range of 400-510 $\mathrm{nm}$ was observed, and the GSB band is centered at $560 \mathrm{~nm}$, which agrees well with the UV-Vis absorption spectra (both in wavelength and width. Figure 2a). The triplet state lifetime was determined as $\tau_{\mathrm{T}}=132.3 \mu \mathrm{s}$, by monitoring the decay trace at $560 \mathrm{~nm}$ (Figure $5 \mathrm{~b}$ ). With fitting of the decay trace based on the kinetics model where triplet-triplet-annihilation (TTA) effect was taken into account, ${ }^{74}$ the intrinsic triplet state of BDP-B was determined as $149 \mu \mathrm{s}$ (Supporting Information, Figure S37) which is similar to the apparent lifetime (132.3 $\mu$ s). This
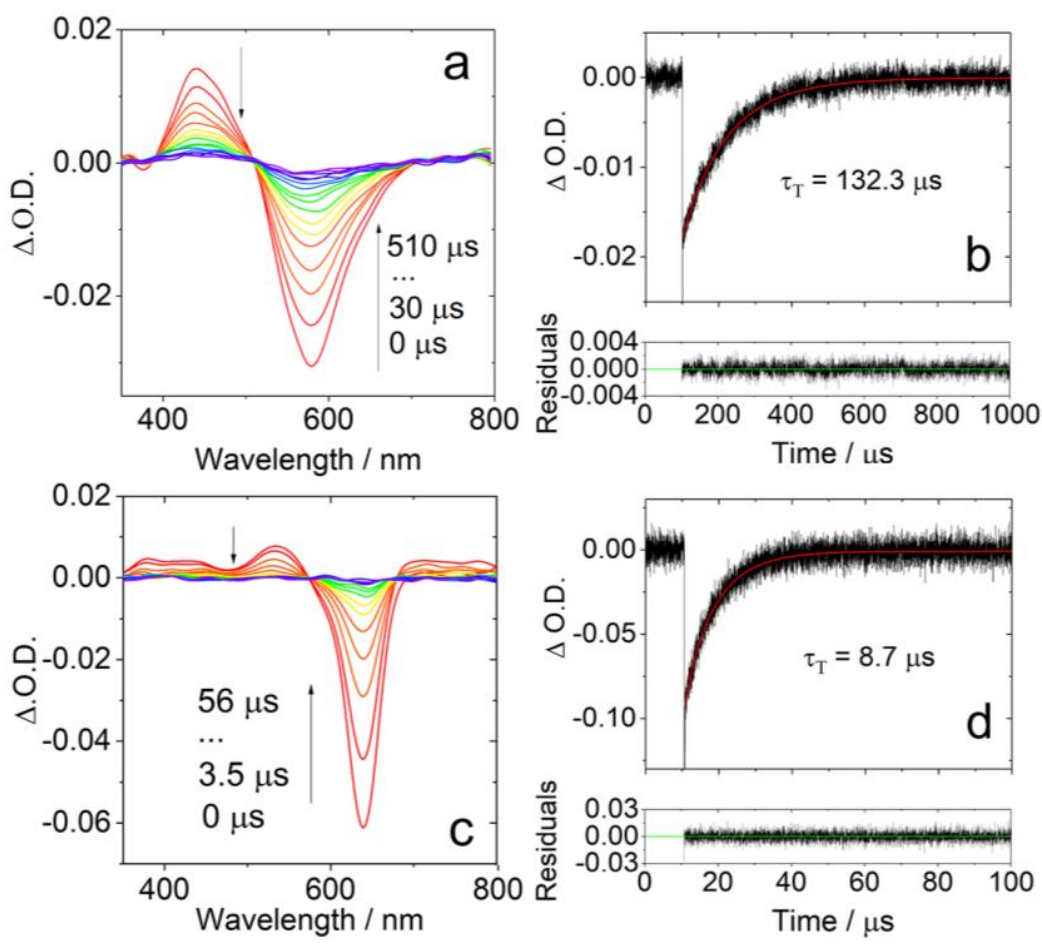

Figure 5. Nanosecond transient absorption spectra of (a) BDP-B in deaerated TOL and (c) BDP-P in deaerated ACN (with 15\% iodoethane added, v/v) at different delay time. (b) Decay traces of BDP-B at $560 \mathrm{~nm}$. $\lambda_{\mathrm{ex}}=572 \mathrm{~nm}$. In deaerated TOL. Decay traces of (d) BDP-P at $640 \mathrm{~nm} . \lambda_{\mathrm{ex}}=625 \mathrm{~nm}$. In deaerated ACN (with $15 \%$ iodoethane added, v/v). $c=1.0 \times 10^{-5} \mathrm{M}$, $20{ }^{\circ} \mathrm{C}$. 
triplet state lifetime is shorter than that observed in 2,6-diiodoBODIPY $(276 \mu$ s $),{ }^{74}$ or in some BODIPY-derived electron donor/acceptor dyads (up to $345 \mu \mathrm{s}$ ), ${ }^{43}$ twisted BDP-N (492 $\left.\mu \mathrm{s}\right),{ }^{58}$ but it is similar to the recently reported twisting BDP-D $(197.5 \mu \mathrm{s}) .^{57}$

Interestingly, the ESA band of BDP-P can only be observed in the presence of iodoethane, indicating weaker ISC for this compound, although it is more twisted than BDP-B. ESA bands in the range of $360-570 \mathrm{~nm}$ and $680-800 \mathrm{~nm}$ were observed, this last band seem to have the same dynamics as the other one, but on the base of the TREPR data (see later section) it might belong to a different triplet state. The GSB band is centered at $640 \mathrm{~nm}$, which is in agreement with the steady-state UV-Vis absorption band (Figure 2b). The triplet state lifetime was measured in the presence of different amounts of iodoethane in ACN (Figure S26). We observed that the proportion of iodoethane has little impact on the triplet state lifetime (ca. 9 $\mu$ ) of BDP-P. For BDP-B, however, the higher the proportion of iodoethane, the shorter the triplet state lifetime $(14.9 \mu \mathrm{s} \sim 30.0 \mu \mathrm{s}$. Figure S26).

Intermolecular triplet-triplet energy transfer (TTET) was used to determine the energy of the $\mathrm{T}_{1}$ state of BDP-B and BDP-P (Supporting Information, Figure S27, S29 and S30). For BDP-B, Zinc phthalocyanine $(\mathrm{ZnPc})\left(E_{\mathrm{T} 1}=1.13 \mathrm{eV}\right)^{96}$ was used as the sensitizer (Supporting Information, Figure S27). TTET was observed, and the triplet state lifetime of ZnPc was reduced from $217 \mu$ s to $80 \mu \mathrm{s}$. The GSB band of BDP-B at $590 \mathrm{~nm}$ firstly grows $(18.8 \mu \mathrm{s})$ then decays $(117.2 \mu \mathrm{s})$, indicating that the $E_{\mathrm{T} 1}$ of BDP-B is lower than $1.13 \mathrm{eV}$. On the other hand, since the BDP-B can sensitize singlet oxygen, the triplet state energy level of BDP-B should 
be higher than $0.98 \mathrm{eV}$. The localization of $\mathrm{T}_{1}$ between 0.98 and $1.13 \mathrm{eV}$ is perfectly consistent with the theoretically calculated value of $1.03 \mathrm{eV}$ (vide infra). For BDP-P, methylene blue (MB) $\left(E_{\mathrm{T} 1}=1.44 \mathrm{eV}\right)$ and 2,6-diiodo BODIPY (diiodo-BDP) $\left(E_{\mathrm{T} 1}=1.61 \mathrm{eV}\right)$ was used as the sensitizer (Figure S29 and S30). ${ }^{96-98}$ With similar TTET studies, we determined that the triplet state energy of BDP-P is in the range of 1.44-1.61 eV. This estimate is again fully consistent with the theoretical value of $1.45 \mathrm{eV}$ (vide infra). The EAS obtained from TTET process in ACN is shown in Figure S31.

The triplet state lifetime of BDP-P was determined as $18.9 \mu$ s by TTET. The triplet state lifetime of BDP-P $\left(\tau_{\mathrm{T}}=8.7 \mu\right.$ s in presence of iodoethane, or $18.9 \mu$ s by TTET $)$ is much shorter than that of BDP-B $\left(\tau_{\mathrm{T}}=132.3 \mu \mathrm{s}\right)$. This is unlikely due to the energy gap law, since the $\mathrm{T}_{1}$ of BDP-B $(0.98 \mathrm{eV}-1.13 \mathrm{eV})$ is significantly lower than BDP-P $(1.44 \mathrm{eV}-1.61 \mathrm{eV})$ counterpart. Interestingly, although the $\mathrm{S}_{1}$ state of BDP-B is short-lived, its $\mathrm{T}_{1}$ state is long-lived. This is another example that the deactivation of the $S_{1}$ and $T_{1}$ states do not follow the same rules. ${ }^{74,99}$ For the occurring of an electronic transition, the molecular vibrational coupling, the molecular orbital integral, the electron spin integral, and the Franck-Condon factor all contribute to the transition probability, making the final effect hard to be intuited. ${ }^{18}$

We underline that BDP-P bears a strong absorption in the red spectral region $\left(\varepsilon=8.7 \times 10^{4}\right.$ $\mathrm{M}^{-1} \mathrm{~cm}^{-1}$ at $\left.635 \mathrm{~nm}\right)$, yet the $\mathrm{T}_{1}$ state energy is high $(1.44-1.61 \mathrm{eV})$, similar to the native BODIPY chromophore $(1.7 \mathrm{eV})$, which has an absorption band centered at ca. $500 \mathrm{~nm} .{ }^{97,98,100}$ The reason for these trend is attributed to the different spatial confinement of the singlet excited 
state wave function and the triplet excited state wave function. For the former, it is likely more delocalized, consistent with the red-shifted absorption, whereas the latter is more confined (supported by the later TREPR analysis) and the spin density surface of the $\mathrm{T}_{1}$ state, consistent with a relatively high $\mathrm{T}_{1}$ energy. This aspect is also discussed with the help of our calculations below. This may be a key aspect in the molecular design method of PSs. ${ }^{101}$

The triplet state properties of the compounds doped in the Clear Flex $50^{\circledR}$ film ${ }^{102}$ were studied (Figure S31), because it is known that the matrix may exert substantial impact on the triplet state property. ${ }^{97}$ For BDP-B, the ESA bands centered at $440 \mathrm{~nm}$ were observed, which are same as the characteristic signals in fluid solutions. Slightly increased $\tau_{\mathrm{T}}(172 \mu \mathrm{s})$ of BDPB in the film was observed, as compared to the $\tau_{\mathrm{T}}(132 \mu \mathrm{s})$ obtained in solution (TOL). It is worth mentioning that the characteristic ESA band of BDP-P was observed in the film, and the triplet lifetime $(87 \mu \mathrm{s})$ is much longer than the triplet lifetime $(8.7 \mu \mathrm{s})$ in solution (ACN with adding $15 \%$ iodoethane).

\subsection{Time-Resolved Electron Paramagnetic Resonance (TREPR) Spectroscopy:}

\section{Confinement of the Triplet State Wave Function and the Electron Spin Selectivity of the}

ISC. To unravel the ISC mechanism in twisted BODIPY derivatives, we studied the electron spin selectivity of the ISC, i.e., the selective population of the three non-degenerate sublevels of the $T_{1}$ triplet state $\left(T_{x}, T_{y}\right.$ and $\left.T_{z}\right)$, and the spatial confinement of the triplet state wave function, the TREPR spectra of the two compounds in frozen solution at $84 \mathrm{~K}$ have been recorded (Figure 6). ${ }^{103-105}$ Magnetophotoselection (MPS) experiments were performed by 
excitation of the samples with laser light polarized perpendicular or parallel to the magnetic field (Figure 6). In a collection of randomly oriented molecules, a polarized light beam selects particular orientations, ${ }^{106}$ and this affects the shape of the EPR spectrum.
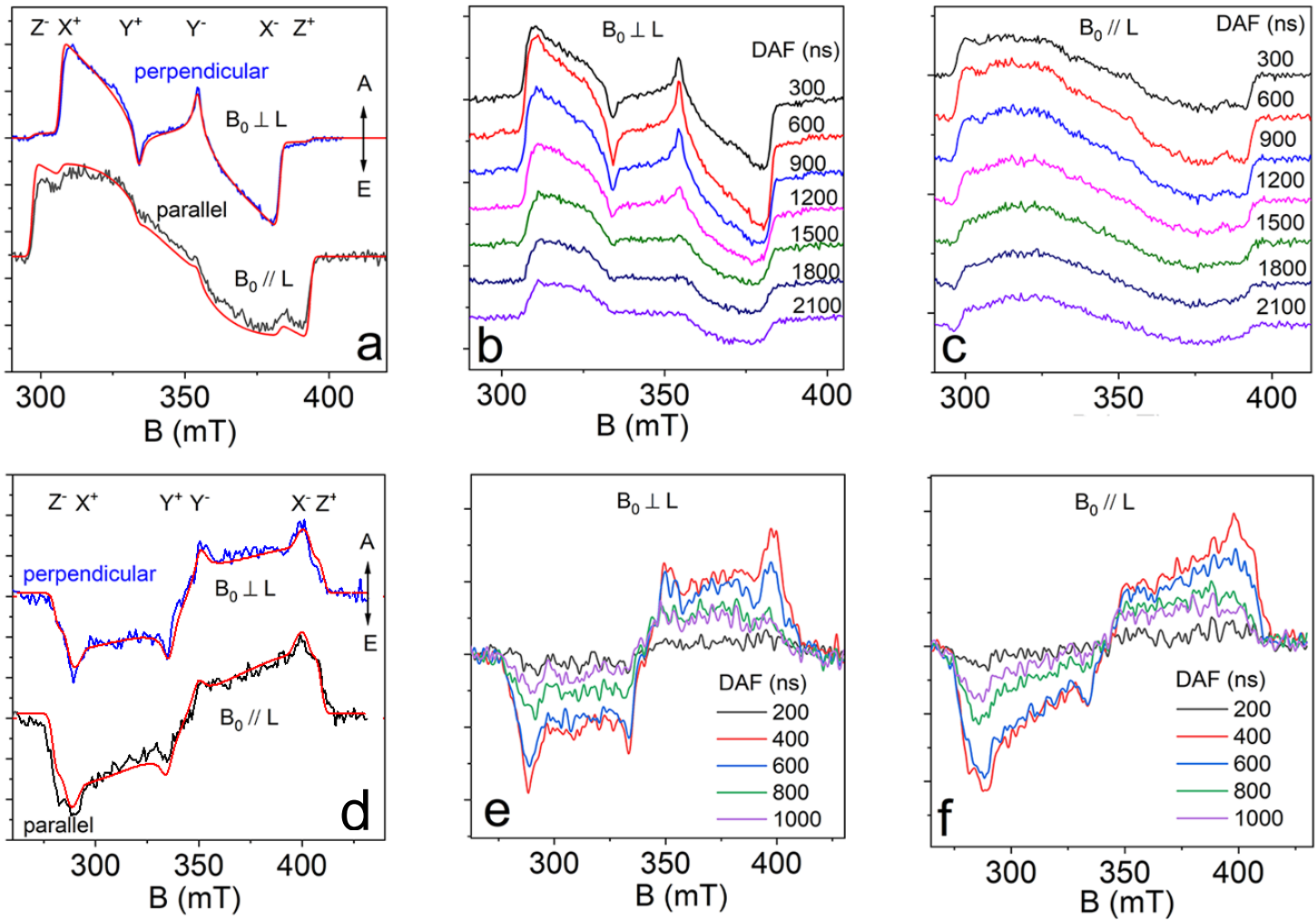

Figure 6. TREPR spectra of (a) BDP-B $\left(\lambda_{\mathrm{ex}}=570 \mathrm{~nm}, c=5.0 \times 10^{-4} \mathrm{M}\right)$ in frozen mixed solution of TOL/MeTHF $(1 / 2, \mathrm{v} / \mathrm{v}$,) for laser polarized perpendicular or parallel to the magnetic field, $T=84 \mathrm{~K}$, DAF (delay after flash) $=600 \mathrm{~ns}$; (b) BDP-B of different DAF for light polarized perpendicular and (c) parallel to the magnetic field. TREPR spectra of (d) BDP-P $\left(\lambda_{\mathrm{ex}}=590 \mathrm{~nm}, c=5.0 \times 10^{-4} \mathrm{M}\right.$. in TOL/MeTHF (1:2, v/v) frozen mixed solution (with $20 \%$ iodoethane added) for light polarized perpendicular or parallel to the magnetic field for DAF = $400 \mathrm{~ns}, 84 \mathrm{~K}$. of (e) BDP-P for different DAF for light polarized perpendicular and (f) parallel to the magnetic field. The fitting parameters are presented in Table 2. The red curves are simulations of the experimental TREPR spectra (black curves and blue curves). In this work we assumed $\mathrm{Z}>0$ and $\mathrm{X}<\mathrm{Y}<\mathrm{Z}$ (see text). 
Previously, a method for the simulation of the TR-EPR spectra obtained with polarized light excitation was developed, based on the assumption that the transition dipole moment (TDM) of the $\mathrm{S}_{0} \rightarrow \mathrm{S}_{1}$ is oriented along one of the principal axes of the zero-field splitting. ${ }^{84}$ Recently this method was presented for arbitrary orientations of the TDM with respect to the principal axes of the zero-field splitting. ${ }^{107}$ The fitting of the TR-EPR spectra in this method required the use of a population distribution function for the photoexcited triplets which included explicitly the orientation of the optical TDM given by $\omega, \phi$ angles in the molecular frame determined by the ZFS tensor (Supporting Information, Figure S35). Therefore, the use of linearly polarized laser light for excitation makes it possible to determine the mutual orientation of TDM and the principal axes of ZFS tensor from the fitting of experimental spectra. The analysis of the TREPR spectra of BDP-B and BDP-P by excitation of the samples with laser light polarized perpendicular or parallel to the magnetic field was carried out using this technique. The simulated best-fit spectra are shown in Figure 6a and $6 \mathrm{~d}$ by red lines. For BDP-B, the overall polarization in the electron spin polarization (ESP) patterns is (AAEAEE) (Figure 6a), where A denotes enhanced absorption and $\mathrm{E}$ denotes emission. This phase polarization pattern is different from the 2,6-diiodoBODIPY and the 2-iodoBodipy, for which the triplet states are formed via ISC in the presence of an heavy atom effect (spin-orbit coupling), the ESP patterns of the triplet state TREPR spectrum being (EEEAAA). ${ }^{43,107}$ The ESP of the triplet state TREPR spectrum of BDP-B differs also from a recently reported twisted BODIPY derivative, which showed a (AEAEAE) ESP pattern. ${ }^{58}$ The ZFS $|D|$ parameter of the triplet state of BDP-B was 
determined as $1350 \mathrm{MHz}$ by simulation, and the $|E|$ value was determined as $260 \mathrm{MHz}$ (Table 2). In comparison, 2-iodoBodipy gives $D$ and $E$ parameters as $-2595 \mathrm{MHz}$ and $575 \mathrm{MHz},{ }^{107}$ respectively. For 2,6-diiodoBODIPY, the $D$ and $E$ parameters are $-2962 \mathrm{MHz}$ and 655 $\mathbf{M H z},{ }^{43,108}$ respectively. These results indicate that the triplet state wave function of BDP-B is located on a larger $\pi$-conjugated system than that of native BODIPY derivatives. For the recently reported BDP-N (Scheme 1), ${ }^{58,86}$ the $D$ and $E$ values are interestingly smaller in magnitude, $-1667 \mathrm{MHz}$ and $308 \mathrm{MHz}$, respectively. ${ }^{58}$ Obviously, the $\pi$-conjugated framework of BDP-B is smaller than the $\pi$-conjugated framework of BDP-N, so one would expect in principle a larger ZFS $D$ for BDP-B, but the reverse is observed. These results indicate that the magnitude of the $D$ values does not simply manifest the extent of confinement of the $\mathrm{T}_{1}$ state wave function. In other words, the triplet state wave function is not systematically delocalized over the whole $\pi$-conjugated framework of the molecule. ${ }^{103}$

The analysis of the TREPR data does not allow determining the sign of $D$. Depending on the sign of $D$, the spectra are described by different population rates of $P_{\mathrm{X}, \mathrm{Y}, \mathrm{Z}}$. Regardless of the sign of $D$, the spectrum shape of BDP-B demonstrates a significant difference from 2iodoBodipy and 2,6-diiodoBodipy. We consider a variant $D<0$ by analogy with 2iodoBodipy $^{107}(D=-2595 \mathrm{MHz})$ and 2,6-diiodoBodipy for which $D=-2962 \mathrm{MHz} \cdot{ }^{43,108}$ Since the ESP of BDP-B is different from that of 2,6-diiodoBODIPY, the selective population of the sublevels of the $\mathrm{T}_{1}$ state is different. For BDP-B, the $\mathrm{T}_{\mathrm{x}}$ sublevel is overpopulated $\left(P_{\mathrm{x}}: P_{\mathrm{y}}: P_{\mathrm{z}}\right.$ $=1.00: 0: 0)$. This is drastically different from 2,6-diiodoBODIPY $\left(P_{\mathrm{x}}: P_{\mathrm{y}}: P_{\mathrm{z}}=0: 0.15:\right.$ 
1.00). ${ }^{43}$ This difference is probably due to the lower symmetry of the molecule, as was observed for twisted Bodipy derivatives BDP-N. ${ }^{58}$ It should be pointed out that for normal $\pi$-conjugated organic compounds with oblate $\mathrm{T}_{1}$ wave function distribution, the triplet state is characterized by a ZFS parameter $D>0$ (for molecules with prolate $\mathrm{T}_{1}$ state wave function distribution, $D<$ $0) .{ }^{103,109-111}$ We found BODIPY chromophore is an exception to this general trend. ${ }^{107}$

For pristine BDP-P, no TREPR signal was detected in frozen solution. When $20 \%$ iodoethane (v/v) was added, the obtained TREPR spectra shows a (EEEAAA) ESP pattern (Figure 6d, black line). The observed pattern of BDP-P is similar with the ESP pattern in 2,6diiodoBodipy while differing from that of BDP-B (AAEAEE), or of twisted BODIPY derivatives showing ISC, e.g., BDP-N (AEAEAE), ${ }^{58}$ and BDP-D (AAEAEE). The influence of iodoethane on the signal of BDP-B was checked (Figure S34), and we found that the impact of the external heavy atom effect on the polarization pattern is negligible. On the other hand, the presence of a triplet produced by charge recombination $(\mathrm{CT})^{112}$ in the spectrum was identified (about $2 / 3$ the intensity of that produced by ISC). A remarkable simulation has been obtained at first from the simulation of the TREPR with isotropic light irradiation (Figure S36), which do not depend on the direction of the TDM's. ${ }^{113}$ The ESP of the CT triplet (Figure S36b) derives from the polarization of the high-field Zeeman states; charge transfer state have been already reported for molecules with similar structures. ${ }^{114}$ Possibly, the CT-formed triplet state is responsible for the transient absorption band at about $700 \mathrm{~nm}$ (see Figure 5 and Figure S30). The ZFS parameters account for a not very different electron distribution with respect to the 
ISC-populated one, having a decrease of the $D$-value by less than $10 \%$. In this work we are mostly interested on the ISC-formed triplet state.

Table 2. Zero-Field Splitting Parameters $(D \text { and } E)^{a}$ and Relative Populations $P_{\mathrm{x}, \mathrm{y}, \mathrm{z}}{ }^{a}$ of the Spin States at Zero Magnetic Field of the Compounds.

\begin{tabular}{ccccccc}
\hline & $D(\mathrm{MHz})$ & $E(\mathrm{MHz})$ & $P_{\mathrm{x}}$ & $P_{\mathrm{y}}$ & $P_{\mathrm{z}}$ & $\mathrm{TDM}(\omega, \phi)^{b}$ \\
\hline BDP-B & -1350 & 260 & 1.00 & 0 & 0 & $\left(10^{\circ}, 90^{\circ}\right)$ \\
BDP-P & -1820 & 467 & 0.23 & 0.00 & 0.77 & $\left(30^{\circ}, 45^{\circ}\right)$ \\
& -1647 & 420 & $P_{+}=1^{c}$ & $P_{0}=0^{c}$ & $P_{-}=1^{c}$ & $\left(30^{\circ}, 45^{\circ}\right)$
\end{tabular}

${ }^{a}$ Obtained from simulations of the triplet-state TREPR spectra of the indicated molecules at $84 \mathrm{~K} . P_{i}$ is the relative population of the $i$-th ZFS state, labeled as indicated in Figure 8. An isotropic g-value of 2.002 was chosen for the spectral simulations of two molecules. ${ }^{b}$ The values were calculated according to the method described in literature. ${ }^{97}$ The orientation of the transition dipole moment in ZFS frame are shown in Supporting Information, Figure S35. ${ }^{c}$ These population values are relative to polarization of high-field Zeeman states.

The ZFS $|D|$ and $|E|$ parameters of BDP-P were determined as $1820 \mathrm{MHz}$ and $467 \mathrm{MHz}$, respectively (Table 2). This is an interesting result because the $|D|$ value is larger than in BDPB (1350 MHz), although BDP-P has apparently a larger $\pi$-conjugated scaffold. The computed spin density surface of the $T_{1}$ state (vide infra) consistently indicates that the triplet state wave function in BDP-P is actually more confined than in BDP-B. For the recently reported BDP$\mathbf{N}$, the $D$ is $-1667 \mathrm{MHz} .{ }^{58}$ For BDP-D, ${ }^{114}$ which is with smaller $\pi$-conjugated framework than BDP-P, the $D$ and $E$ value of the triplet state was determined as $-1820 \mathrm{MHz}$ and $420 \mathrm{MHz}$, respectively. 


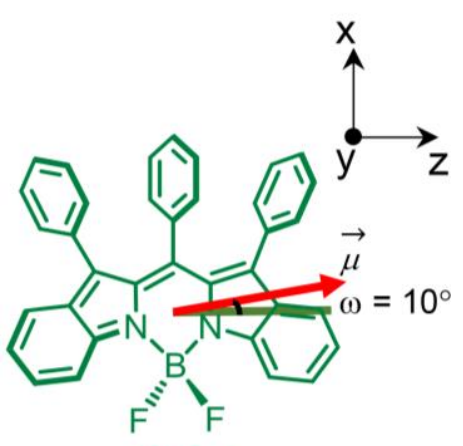

BDP-B

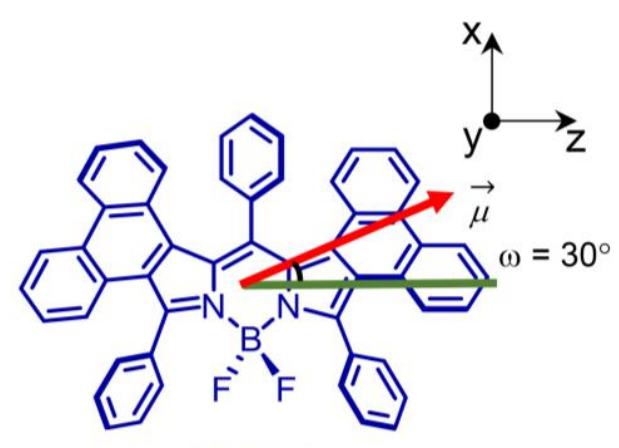

BDP-P

Figure 7. Molecular structures of BDP-B and BDP-P with the principal molecular directions determined by the ZFS tensor. The principal molecular directions also conveniently define the molecular frame. The $\mathrm{S}_{0} \rightarrow \mathrm{S}_{\mathrm{x}}(\mathrm{x}=1,3)$ transition dipole moment $\vec{\mu}$ is drawn in red.

Simulations show that the triplet states in BDP-P have the $\mathrm{T}_{\mathrm{z}}$ state overpopulated $\left(P_{\mathrm{x}}: P_{\mathrm{y}}\right.$ : $P_{\mathrm{z}}=0.23: 0.00: 0.77$, with $D<0$, Table 2 ). This selectivity is drastically different from the ones of BDP-B (vide supra), and BDP-D $\left(P_{\mathrm{x}}: P_{\mathrm{y}}: P_{\mathrm{z}}=0: 1.0: 0.21\right) .{ }^{114}$

The strong intensity of the Z-components when excitation is parallel for these two molecules leads us to infer that the TDM is almost parallel to the Z-principal direction of the triplet state (Figure 6 and Table 2: tilt angles of ca. 10 and $30^{\circ}$ ). ${ }^{107,115}$ The orientation of the ZFS principal axes with respect to the TDM were determined as $10^{\circ}$ and $30^{\circ}$ for BDP-B and BDP-P, respectively, with a previously reported method. ${ }^{107} \mathrm{We}$ note that with proper orientation of the phenyl groups, the BDP-B and BDP-P molecules have $\mathrm{C}_{2 \mathrm{v}}$ symmetry, therefore we expect the ZFS principal axes and the TDM to be oriented along the symmetry axes. Instead, both molecules have a mismatch between the Z-spin dipolar axis and the TDM; this can be 
interpreted as a clear distortion of the molecule from this symmetry, and the distortion relative to BDP-P is larger, at least in terms of electron density.

\subsection{Theoretical Computations: Rationalization of the Photophysical Properties of the}

Compounds. Due to the cyanine character of BODIPY chromophore, TD-DFT methods are known to lead to especially large errors ${ }^{116}$ (above the usual ca. $0.3 \mathrm{eV}$ error bar of TD-DFT) for study of BODIPY compounds. ${ }^{117}$ We have therefore chosen to use a post-HF computational method for these compounds, namely the second-order Algebraic Diagrammatic Construction, Table 3 Electronic Excitation Energies (eV) and Corresponding Oscillator Strengths ( $f$ ) for BDP-P. ${ }^{a}$

\begin{tabular}{|c|c|c|c|c|c|c|c|}
\hline \multirow[b]{2}{*}{ Transition } & \multicolumn{3}{|c|}{$\operatorname{ADC}(2) / c c-p V D Z$} & \multicolumn{3}{|c|}{ ADC(2)/aug-cc-pVDZ } & \multirow[b]{2}{*}{$\lambda \exp$} \\
\hline & $\Delta \boldsymbol{E}_{\mathbf{t h}}{ }^{a}$ & $f^{c}$ & $\lambda_{\text {th }^{d}}$ & $\Delta \boldsymbol{E}_{\mathbf{t h}}^{b}$ & $f^{c}$ & $\lambda_{\text {th }^{d}}$ & \\
\hline $\mathrm{S}_{0} \rightarrow \mathrm{S}_{1}$ & 2.021 & 0.78 & 614 & 1.966 & 0.74 & 630 & $635^{e}$ \\
\hline $\mathrm{S}_{0} \rightarrow \mathrm{S}_{2}$ & 2.594 & 0.16 & 478 & 2.531 & 0.15 & 490 & \\
\hline $\mathrm{S}_{0} \rightarrow \mathrm{S}_{3}$ & 2.655 & 0.06 & 467 & 2.594 & 0.06 & 478 & \\
\hline $\mathrm{S}_{0} \rightarrow \mathrm{S}_{4}$ & 3.064 & 0.01 & 405 & 2.978 & 0.01 & 416 & \\
\hline $\mathrm{S}_{0} \rightarrow \mathrm{S}_{5}$ & 3.215 & 0.01 & 386 & 3.129 & 0.01 & 396 & \\
\hline $\mathrm{S}_{1} \rightarrow \mathrm{S}_{0}$ & 1.378 & 0.36 & 899 & $1.323^{g}$ & $0.33^{g}$ & $937^{\mathrm{g}}$ & $670^{f}$ \\
\hline
\end{tabular}

${ }^{a}$ GS geometry optimized at the MP2/cc-pVDZ level in gas phase, $\mathrm{S}_{1}$ geometry optimized at the $\mathrm{ADC}(2) / \mathrm{cc}-\mathrm{pVDZ}$ level in gas phase. ${ }^{b}$ Stands for the calculated energy of the transition in $\mathrm{eV}$. ${ }^{c}$ Oscillator strength associated to the corresponding transition. ${ }^{d}$ Stands for the calculated energy of the transition in $\mathrm{nm}$. ${ }^{e} \lambda^{\exp }{ }_{\text {abs, max }}$ recorded in acetonitrile. In $\mathrm{nm}$. ${ }^{f} \lambda{ }^{e x p}$ em, max recorded in acetonitrile in $\mathrm{nm} .{ }^{g}$ Theses values were computed using the cc-pVDZ basis-set for hydrogens and aug-cc-pVDZ for all heavy atoms, i.e., except hydrogens. This choice was made because the addition of diffuse on the $\mathrm{H}$ prevents the calculation to properly converge. 


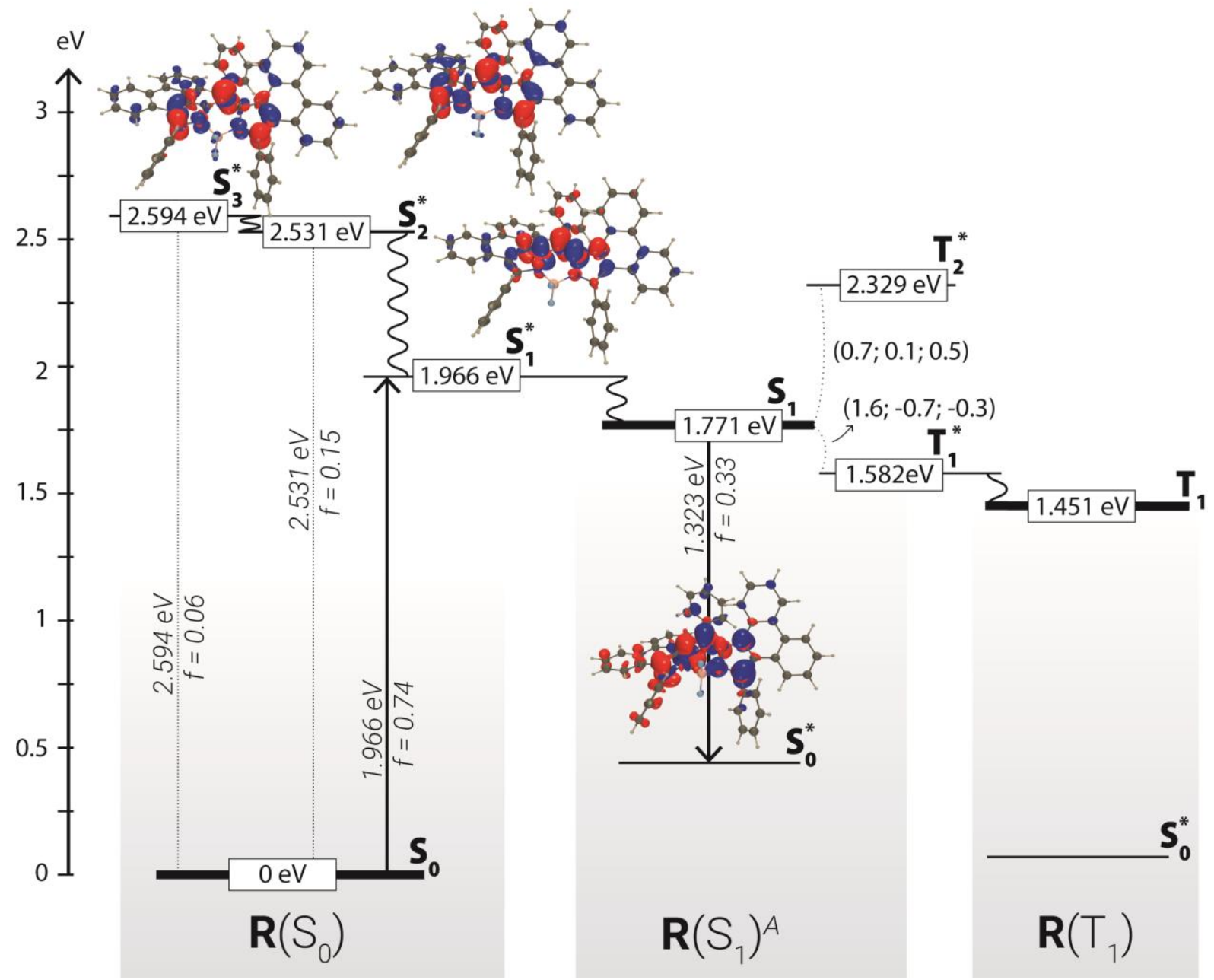

Figure 8. Jablonski diagram of BDP-P including EDD plots for the corresponding vertical transition. Energies were calculated at the ADC(2)/aug-cc-pVDZ level on MP2 or ADC(2)/ccpVDZ optimized geometries. SOCs $(x ; y ; z)$ components are given in $\mathrm{cm}^{-1}$ and obtained with QR-TD-B3LYP/6-31G. EDD isovalues $=0.0015$ au. The red and blue lobes of the EDD indicate increase and decrease of the electron density upon absorption, respectively. Theses energies are computed using the cc-pVDZ basis-set on hydrogens and aug-cc-pVDZ for all heavy atoms ie. except hydrogens. 


\begin{abstract}
ADC(2), approach. ${ }^{118}$ On the other hand, as the studied compounds do not exhibit large solvatochromism neither for absorption nor for emission, our simulations were made in gas phase, for the sake of saving computational time. Our results are displayed in Tables 3 and 4, as well as Figures 8 and 9.
\end{abstract}

The experimental absorptions values are very well reproduced $\left(0.01 \mathrm{eV}<\left|\Delta E_{\text {exp-th }}\right|<0.07\right.$ eV) for BDP-P, which exhibits a classical BODIPY behavior, with a strongly dipole-allowed $\mathrm{S}_{0} \rightarrow \mathrm{S}_{1}$ transition $(f=0.74)$ (see Table 3 ), for which the electron density difference (EDD) plots (Figure 8) shows a loss of electronic density on the non-meso carbons of the six membered cycle of the BODIPY core along with a gain of density on the meso carbon atom and the nitrogen atoms bonded to the boron, a very usual excited state topology for a BODIPY derivative. ${ }^{119}$ Following relaxation of the geometry of the $\mathrm{S}_{1}$, there can be of course fluorescence for which the theory predicts a low value, which is a usual error sign for ADC(2) in that spectra region. More importantly, only one triplet state is accessible from the $\mathrm{S}_{1}$ minimum, and ISC can only take place to that state (see also the computed SOCs in Figure 8). The computed $\mathrm{T}_{1}$ is located at $1.58 \mathrm{eV}$ ( $\mathrm{S}_{1}$ geometry) or $1.45 \mathrm{eV}$ ( $\mathrm{T}_{1}$ geometry), a value consistent with the 1.44-1.61 eV window provided by experiments. In short, BDP-P behaves in a rather "classical" BODIPY way with respect to the photophysical property. The magnitude of the calculated spin orbital coupling matrix elements (SOCME) between the $\mathrm{S}_{1}$ and $\mathrm{T}_{1}{ }^{*}$ states is $1.81 \mathrm{~cm}^{-1}$. However, negligible ISC was observed for BDP-P, which may be attributed to the symmetry forbidden feature of the $\mathrm{S}_{1} \rightarrow \mathrm{T}_{1}$ ISC. 


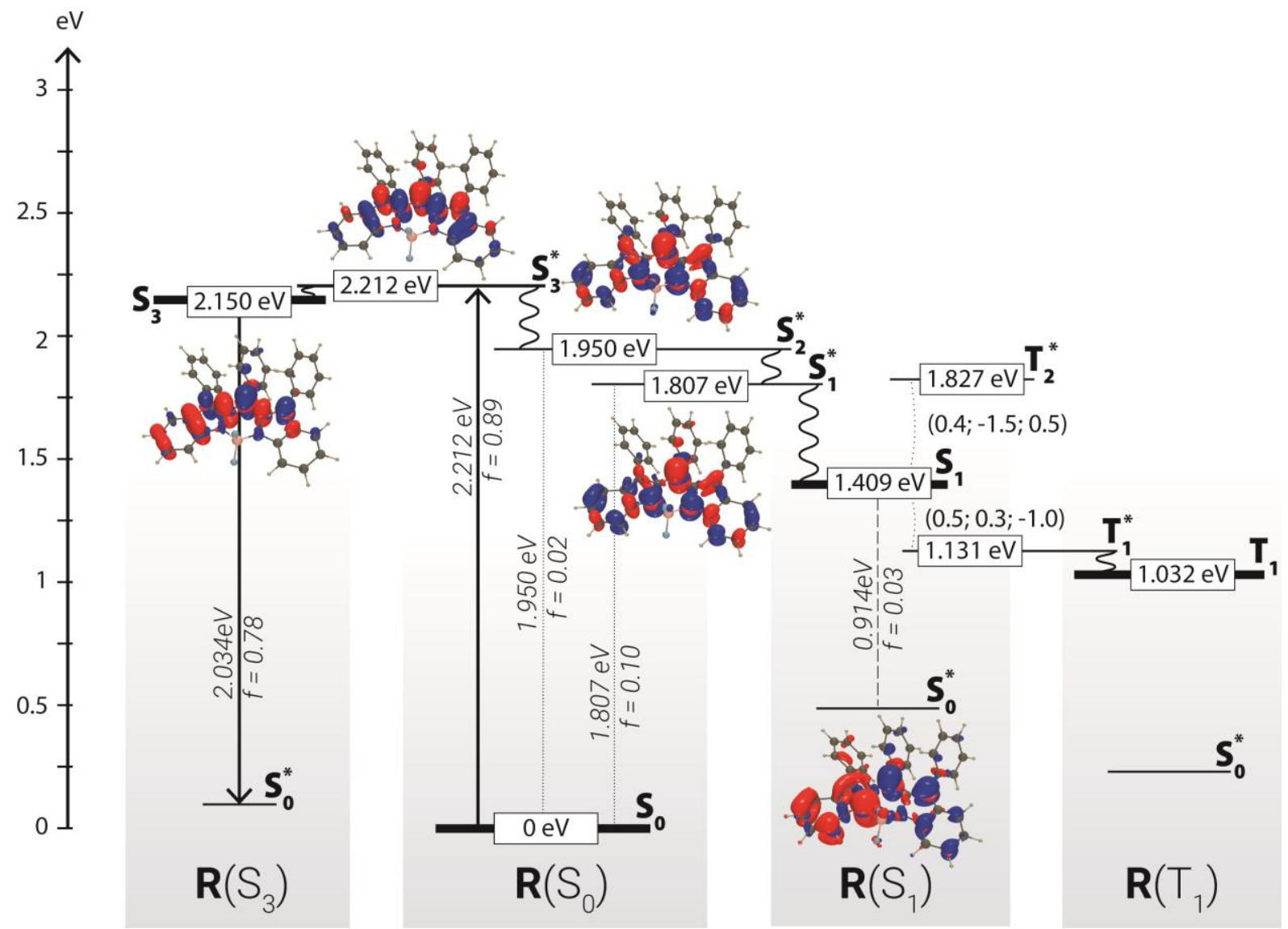

Figure 9. Jablonski diagram of BDP-B including EDD plots for the corresponding vertical

transition. $\mathrm{S}_{0}$ geometry optimized at MP2/cc-pVDZ, $\mathrm{S}_{1}, \mathrm{~S}_{3}$ and $\mathrm{T}_{1}$ geometries optimized at $\operatorname{ADC}(2) / c c-p V D Z$, energies in eV computed at the ADC(2)/aug-cc-pVDZ level, SOCs (x; y; $z)$ components are given in $\mathrm{cm}^{-1}$ and obtained with QR-TD-B3LYP/6-31G*, all computations were performed in gas phase. EDD isovalues $=0.0015 \mathrm{au}$. The red and blue lobes of the EDD indicate increase and decrease of the electron density upon absorption, respectively. On the left-hand part is the less probable process regarding the low experimental fluorescence quantum yield. 
In contrast, BDP-B does not exhibit a traditional BODIPY behavior as the $\mathrm{S}_{0} \rightarrow \mathrm{S}_{1}$ transition is forbidden, it presents a very small oscillator strength with aug-cc-pVDZ $(f=0.10$, see Table 4), whereas the $S_{1} \rightarrow S_{3}$ transition seems to contribute to the main absorption band centered at $569 \mathrm{~nm}(561 \mathrm{~nm}$ by computation) with a large oscillator strength $(f=0.89)$. We note that the topology of the experimental absorption band indeed departs from the usual narrow and sharp peak with a vibronic shoulder of most BODIPYs, consistent with the theoretical result. At the optimized $\mathrm{S}_{1}$ geometry, that is for the putative "emissive" structure, one obtains a ridiculously low $\Delta E\left(E_{\mathrm{S} 0}-E_{\mathrm{S} 1}\right)$ at $0.943 \mathrm{eV}$ with a negligible oscillator strength $(f=0.04)$. Therefore, one clearly expects the $S_{1}$ state relaxes to the ground-state non-adiabatically, owing to strong vibronic coupling that should be dominant for such low-lying dark state. When looking at the EDD plots for the three first transitions in BDP-B at the GS geometry (Figure 9), it is clear that both the $\mathrm{S}_{0} \rightarrow \mathrm{S}_{1}$ and $\mathrm{S}_{0} \rightarrow \mathrm{S}_{2}$ transitions present the same characteristics with a strong loss of density at the nitrogen atoms upon absorption, whereas the topology of the $\mathrm{S}_{0} \rightarrow \mathrm{S}_{3}$ transitions is highly similar to the $\mathrm{S}_{0} \rightarrow \mathrm{S}_{1}$ transition of BDP-P. The (very weak) residual fluorescence observed experimentally in BDP-B can be attributed to an emission from a relaxed $\mathrm{S}_{1}$ to the $\mathrm{S}_{0}$ (Figure 9). ${ }^{120}$ These theoretical predictions are corroborated by the short fluorescence lifetime of BDP-B (biexponential, a fast decay component probably due to the fast IC from the $\mathrm{S}_{1}$ state to $\mathrm{S}_{0}$ state and a slow decay component due to the residual fluorescence, see Figure 3). In what concerns ISC, $\mathrm{ADC}(2)$ predicts only one triplet below $\mathrm{S}_{1}$, so that the process is clear (see also the computed SOCs in Figure 9). Interestingly, this triplet is located at $1.13 \mathrm{eV}$ ( $\mathrm{S}_{1}$ geometry) 
or $1.03 \mathrm{eV}$ ( $\mathrm{T}_{1}$ geometry), significantly below the BDP-P, and again, in remarkable agreement with the experimentally determined window (vide supra).

Table 4. Electronic Excitation Energies (eV) and Corresponding Oscillator Strengths (f) for BDP-B ${ }^{a}$

\begin{tabular}{|c|c|c|c|c|c|c|c|}
\hline \multirow[b]{2}{*}{ Transition } & \multicolumn{3}{|c|}{$\operatorname{ADC}(2) / c c-p V D Z$} & \multicolumn{3}{|c|}{ ADC(2)/aug-cc-pVDZ } & \multirow[b]{2}{*}{$\lambda \exp$} \\
\hline & $\Delta \boldsymbol{E}_{\mathbf{t h}}^{b}$ & $f^{c}$ & $\lambda_{\text {th }}{ }^{d}$ & $\Delta \boldsymbol{E}_{\mathbf{t h}}^{b}$ & $f^{c}$ & $\lambda_{\mathbf{t h}^{d}}$ & \\
\hline $\mathrm{S}_{0} \rightarrow \mathrm{S}_{1}$ & 1.852 & 0.11 & 669 & 1.807 & 0.10 & 686 & $579^{e}$ \\
\hline $\mathrm{S}_{0} \rightarrow \mathrm{S}_{2}$ & 1.993 & 0.02 & 622 & 1.950 & 0.02 & 636 & \\
\hline $\mathrm{S}_{0} \rightarrow \mathrm{S}_{3}$ & 2.655 & 0.92 & 550 & 2.212 & 0.89 & 561 & \\
\hline $\mathrm{S}_{1} \rightarrow \mathrm{S}_{0}$ & 0.943 & 0.04 & 1315 & 0.914 & 0.03 & 1356 & $747^{f}$ \\
\hline $\mathrm{S}_{3} \rightarrow \mathrm{S}_{0}$ & 2.077 & 0.82 & 597 & 2.034 & 0.78 & 610 & \\
\hline
\end{tabular}

${ }^{a} \mathrm{GS}$ geometry optimized at the MP2/cc-pVDZ level in gas phase, $\mathrm{S}_{1}$ and $\mathrm{S}_{3}$ geometries optimized at the ADC(2)/cc-pVDZ level in gas phase. ${ }^{b}$ Stands for the calculated energy of the transition in $\mathrm{eV} .{ }^{c}$ Oscillator strength associated to the corresponding transition. ${ }^{d}$ Stands for the calculated energy of the transition in $\mathrm{nm}$. ${ }^{e} \lambda^{\exp }{ }_{\mathrm{abs}}$, max recorded in toluene in $\mathrm{nm} .{ }^{f} \lambda^{\mathrm{exp}}{ }_{\mathrm{em}}$, max recorded in toluene in $\mathrm{nm}$.

The magnitude of the computed SOCME of the $\mathrm{S}_{1} / \mathrm{T}_{1}{ }^{*}$ state is $1.14 \mathrm{~cm}^{-1}$, which is significant. This value is even larger than a recently reported twisting BODIPY showing efficient ISC $\left(\Phi_{\Delta}\right.$ $=52 \%$ ), with SOCME of $0.1689 \mathrm{~cm}^{-1}{ }^{58}$ The symmetry of the $\mathrm{S}_{1} / \mathrm{T}_{1}{ }^{*}$ state satisfy the selection rule for ISC. Thus, the moderate ISC ability of BDP-B is rationalized.

To gain more insights into the nature of the triplet states, spin density surfaces of the $T_{1}$ state of the compounds have been studied (Figure 10). Normally, as discussed above, the larger the $\pi$-conjugated framework of the compound, the smaller the $D$ value (manifesting the dipole- 

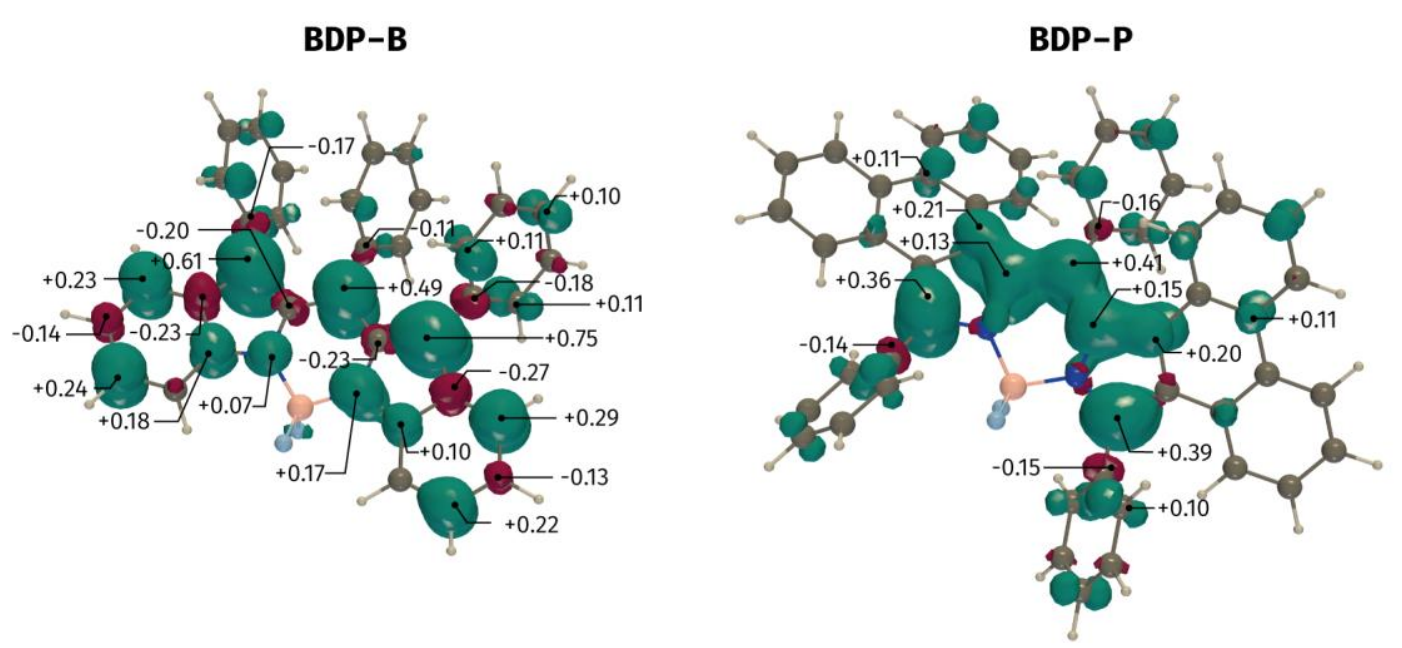

Figure 10. Isosurface of spin density difference $(\alpha-\beta)$ of the $T_{1}$ states of (a) BDP-B and (b) BDP-P. Calculated at UB3LYP/aug-cc-pVDZ level on the ADC(2) geometries. Positives differences are in green, negative in purple, isovalue $=0.0025$ au. The atomic Mulliken spin density population is given for the most spin polarized centers (threshold at $0.1 \mathrm{au}$ ).

dipole interaction magnitude, reciprocal to $r^{3}$, according to point dipole approximation), however, for BDP-P, the $\pi$-conjugated framework is larger than that of BDP-B, but the value of the $D$ of BDP-P is larger, indicating that the triplet state is more confined in BDP-P. Indeed, the triplet state wave function of BDP-B is delocalized on both the BODIPY chromophore and the peripheral fused phenyl rings (Figure 10), whereas in BDP-P, the triplet state wave function is clearly more confined within the dipyrrin core, the distribution of the spin density on the fused phenanthrene being very limited. Thus, the anomalous larger ZFS $D$ parameter and the high $\mathrm{T}_{1}$ state energy of BDP-P are rationalized.

\section{CONCLUSION}


We studied the intersystem crossing (ISC) of two BODIPY derivatives (Benzo[b]-fused BODIPY, BDP-B and $[a]$ Phenanthrene-fused BODIPY, BDP-P), both showing twisted $\pi$ conjugated frameworks, but to different extent. Our aim was to investigate the relationships between the twisting of the $\pi$-conjugation framework of a chromophore and the ISC efficiency. Interestingly, in contrast with the known principles for the ISC of helicene compounds (in which the ISC efficiency depends on the twisting magnitude of the conjugation), the current BODIPY derivatives show counter-intuitive trends: the more twisted structure does not induce higher ISC yield. In addition, BDP-B, the least twisted molecular structure, shows much shorter fluorescence lifetime (ca. $150 \mathrm{ps)}$ ) and much weaker fluorescence $\left(\Phi_{\mathrm{F}}<0.1 \%\right)$ than the most twisted molecule (BDP-P, $\tau_{\mathrm{F}}=6.4 \mathrm{~ns}, \Phi_{\mathrm{F}}=70 \%$ ). ADC(2) calculations show that BDP-B presents a low-lying dark state $\left(f=0.04\right.$ for $\mathrm{S}_{1} \rightarrow \mathrm{S}_{0}$ transition), which is very unusual for BODIPY chromophore, whereas BDP-P behaves more like a classical BODIPY dye $(f=0.33$ for $\mathrm{S}_{1} \rightarrow \mathrm{S}_{0}$ transition). Interestingly, the triplet state lifetime of BDP-B $\left(\tau_{\mathrm{T}}=132.3 \mu \mathrm{s}\right)$ is longer than that of BDP-P $\left(\tau_{\mathrm{T}}=18.9 \mu \mathrm{s}\right.$, measured by photosensitizing method; with addition of iodoethane, the lifetime was determined as $8.7 \mu \mathrm{s}$ ), and this difference cannot be rationalized by the energy gap law, as the $\mathrm{T}_{1}$ state of BDP-B $(0.98-1.13 \mathrm{eV})$ is lower than BDP-P $(1.44-1.61 \mathrm{eV})$. These results indicate that the fast non-radiative decay channel of BDP-B singlet is not working for relaxation of its $\mathrm{T}_{1}$ state. Interestingly, the twisted BDP-P shows the desired photophysical property of red-shifted absorption, yet un-decreased $\mathrm{T}_{1}$ state energy $(1.44-1.61 \mathrm{eV})$ as compared to the native BODIPY (ca. $1.60 \mathrm{eV})$. Time-resolved electron 
paramagnetic resonance (TREPR) spectra show that the confinement of the triplet state wave function of the two compounds are counter-intuitive: BDP-B, with apparently smaller $\pi$ conjugated framework, gives smaller zero-field splitting (ZFS) D parameter $(-1350 \mathrm{MHz})$, whereas the BDP-P, with larger $\pi$-conjugated framework, gives larger D parameter $(-1820$ MHz), normally the opposite holds. These anomalous $D$ parameters of the analogues could be rationalized by the spin density surface of the triplet state of the two compounds: the triplet state wave function of BDP-P is spatially more confined. The phase pattern of the triplet state TREPR spectra of the two compounds are AAEAEE and EEEAAA, both are different from a recently reported twisted BODIPY derivative. These information are useful for in-depth understanding the ISC efficiency of molecules with twisted $\pi$-conjugated framework, and for design of heavy atom-free triplet photosensitizers, as well as for study of the photochemistry of BODIPY chromophores.

\title{
ASSOCIATED CONTENT
}

\section{S Supporting Information}

The Supporting Information is available free of charge on the ACS Publications website at DOI: $10.1021 / \mathrm{xxxx}$

\section{AUTHOR INFORMATION}

\author{
Corresponding Authors \\ *E-mail: zhaojzh@dlut.edu.cn (J. Z.) \\ *E-mail: vio@kfti.knc.ru (V. K V.)
}




\author{
*E-mail: Denis.Jacquemin@univ-nantes.fr (D.J.) \\ * E-mail: xingyongheng2000@163.com (Y.H.X.) \\ * Email: Daniel.escudero@univ-nantes.fr (D.E.) \\ * E-mail: antonio.barbon@unipd.it (A. B.) \\ * Email: gurzadyan@dlut.edu.cn (G.G.G)
}

\title{
Notes
}

The authors declare no competing financial interest.

\section{口 ACKNOWLEDGEMENT}

J.Z. thanks the NSFC (U2001222, 21673031, 21761142005 and 21911530095) and the State Key Laboratory of Fine Chemicals (ZYTS201901) for financial support. A.A.S., and V.K.V. appreciate the support of the Russian Foundation for Basic Research (Project 19-5353013). D. E acknowledges the Research Foundation - Flanders (FWO) and internal KU Leuven funds. M.H.E.B., D.E. and D.J. are indebted to the PHC program QCQY for supporting their collaboration. M.H.E.B. and D.J. are indebted to the CCIPL computational center installed in Nantes for very generous allocation of computational time.

\section{口EFERENCES}

(1) Zhao, J.; Wu, W.; Sun, J.; Guo, S. Triplet Photosensitizers: from Molecular Design to Applications. Chem. Soc. Rev. 2013, 42, 5323-5351.

(2) Zhao, J.; Xu, K.; Yang, W.; Wang, Z.; Zhong, F. The Triplet Excited State of Bodipy: Formation, Modulation and Application. Chem. Soc. Rev. 2015, 44, 8904-8939. 
(3) Sasikumar, D.; John, A. T.; Sunny, J.; Hariharan, M. Access to the Triplet Excited States of Organic Chromophores. Chem. Soc. Rev. 2020, 49, 6122-6140.

(4) Shi, L.; Xia, W. Photoredox Functionalization of C-H Bonds Adjacent to a Nitrogen Atom. Chem. Soc. Rev. 2012, 41, 7687-7697.

(5) Hari, D. P.; König, B. The Photocatalyzed Meerwein Arylation: Classic Reaction of Aryl Diazonium Salts in a New Light. Angew. Chem. Int. Ed. 2013, 52, 4734-4743.

(6) Guo, S.; Chen, K.-K.; Dong, R.; Zhang, Z.-M.; Zhao, J.; Lu, T.-B. Robust and LongLived Excited State Ru(II) Polyimine Photosensitizers Boost Hydrogen Production. ACS Catalysis 2018, 8, 8659-8670.

(7) Majumdar, P.; Nomula, R.; Zhao, J. Activatable Triplet Photosensitizers: Magic Bullets for Targeted Photodynamic Therapy. J. Mater. Chem. C 2014, 2, 5982-5997.

(8) Stacey, O. J.; Pope, S. J. A. New Avenues in the Design and Potential Application of Metal Complexes for Photodynamic Therapy. RSC Adv. 2013, 3, 25550-25564.

(9) Kamkaew, A.; Lim, S. H.; Lee, H. B.; Kiew, L. V.; Chung, L. Y.; Burgess, K. Bodipy Dyes in Photodynamic Therapy. Chem. Soc. Rev. 2013, 42, 77-88.

(10) Li, X.; Kolemen, S.; Yoon, J.; Akkaya, E. U. Activatable Photosensitizers: Agents for Selective Photodynamic Therapy. Adv. Funct. Mater. 2017, 27, 1604053.

(11) Köhler, A.; Bässler, H. Triplet States in Organic Semiconductors. Mater. Sci. Eng. R Rep. 2009, 66, 71-109. 
(12) Dai, F.-R.; Zhan, H.-M.; Liu, Q.; Fu, Y.-Y.; Li, J.-H.; Wang, Q.-W.; Xie, Z.; Wang, L.; Yan, F.; Wong, W.-Y. Platinum(II)-Bis(Aryleneethynylene) Complexes for SolutionProcessible Molecular Bulk Heterojunction Solar Cells. Chem. -Eur. J. 2012, 18, 1502-1511.

(13) Singh-Rachford, T. N.; Castellano, F. N. Photon Upconversion Based on Sensitized Triplet-Triplet Annihilation. Coord. Chem. Rev. 2010, 254, 2560-2573.

(14) Ye, C.; Zhou, L.; Wang, X.; Liang, Z. Photon Upconversion: From Two-Photon Absorption (TPA) to Triplet-Triplet Annihilation (TTA). Phys. Chem. Chem. Phys. 2016, 18, $10818-10835$.

(15) Ceroni, P. Energy up-Conversion by Low-Power Excitation: New Applications of an Old Concept. Chem. -Eur. J. 2011, 17, 9560-9564.

(16) Monguzzi, A.; Tubino, R.; Hoseinkhani, S.; Campione, M.; Meinardi, F. Low Power, Non-Coherent Sensitized Photon up-Conversion: Modelling and Perspectives. Phys. Chem. Chem. Phys. 2012, 14, 4322-4332.

(17) Zhao, J.; Ji, S.; Guo, H. Triplet-Triplet Annihilation Based Upconversion: From Triplet Sensitizers and Triplet Acceptors to Upconversion Quantum Yields. RSC Adv. 2011, 1, $937-950$.

(18) Turro, N. J.; Ramamurthy, V.; Scaiano, J. C. Principles of Molecular Photochemistry: An Introduction, University Science Books, Sausalito, CA, 2009. 
(19) Tilley, A. J.; Pensack, R. D.; Lee, T. S.; Djukic, B.; Scholes, G. D.; Seferos, D. S. Ultrafast Triplet Formation in Thionated Perylene Diimides. J. Phys. Chem. C 2014, 118, 9996-10004.

(20) Pearce, N.; Davies, E. S.; Horvath, R.; Pfeiffer, C. R.; Sun, X.-Z.; Lewis, W.; McMaster, J.; George, M. W.; Champness, N. R. Thionated Naphthalene Diimides: Tuneable Chromophores for Applications in Photoactive Dyads. Phys. Chem. Chem. Phys. 2018, 20, $752-764$.

(21) Nguyen, V.-N.; Qi, S.; Kim, S.; Kwon, N.; Kim, G.; Yim, Y.; Park, S.; Yoon, J. An Emerging Molecular Design Approach to Heavy-Atom-Free Photosensitizers for Enhanced Photodynamic Therapy under Hypoxia. J. Am. Chem. Soc. 2019, 141, 16243-16248.

(22) Hussain, M.; Zhao, J.; Yang, W.; Zhong, F.; Karatay, A.; Yaglioglu, H. G.; Yildiz, E. A.; Hayvali, M. Intersystem Crossing and Triplet Excited State Properties of Thionated Naphthalenediimide Derivatives. J. Luminsc. 2017, 192, 211-217.

(23) Zhao, W.; Castellano, F. N. Upconverted Emission from Pyrene and Di-TertButylpyrene Using $\operatorname{Ir}($ Ppy) 3 as Triplet Sensitizer. J. Phys. Chem. A 2006, 110, 11440-11445.

(24) Specht, D. P.; Martic, P. A.; Farid, S. Ketocoumarins: A New Class of Triplet Sensitizers. Tetrahedron 1982, 38, 1203-1211.

(25) Zhao, Y.; Sukhanov, A. A.; Duan, R.; Elmali, A.; Hou, Y.; Zhao, J.; Gurzadyan, G. G.; Karatay, A.; Voronkova, V. K.; Li, C. Study of the Spin-Orbit Charge Transfer Intersystem Crossing of Perylenemonoimide-Phenothiazine Compact Electron Donor/Acceptor Dyads 
with Steady-State and Time-Resolved Optical and Magnetic Spectroscopies. J. Phys. Chem. C 2019, 123, 18270-18282.

(26) Zhang, X.; Elmali, A.; Duan, R.; Liu, Q.; Ji, W.; Zhao, J.; Li, C.; Karatay, A. Charge Separation, Recombination and Intersystem Crossing of Directly Connected Perylenemonoimide-Carbazole Electron Donor/Acceptor Dyads. Phys. Chem. Chem. Phys. 2020, 22, 6376-6390.

(27) Wang, Z.; Xie, Y.; Xu, K.; Zhao, J.; Glusac, K. D. Diiodobodipy-Styrylbodipy Dyads: Preparation and Study of the Intersystem Crossing and Fluorescence Resonance Energy Transfer. J. Phys. Chem. A 2015, 119, 6791-6806.

(28) Huang, L.; Yu, X.; Wu, W.; Zhao, J. Styryl Bodipy-C60 Dyads as Efficient HeavyAtom-Free Organic Triplet Photosensitizers. Org. Lett. 2012, 14, 2594-2597.

(29) Huang, L.; Zhao, J.; Guo, S.; Zhang, C.; Ma, J. Bodipy Derivatives as Organic Triplet Photosensitizers for Aerobic Photoorganocatalytic Oxidative Coupling of Amines and Photooxidation of Dihydroxylnaphthalenes. J. Org. Chem. 2013, 78, 5627-5637.

(30) Zhao, J.; Ji, S.; Wu, W.; Wu, W.; Guo, H.; Sun, J.; Sun, H.; Liu, Y.; Li, Q.; Huang, L. Transition Metal Complexes with Strong Absorption of Visible Light and Long-Lived Triplet Excited States: From Molecular Design to Applications. RSC Adv. 2012, 2, 1712-1728.

(31) Lincoln, R.; Kohler, L.; Monro, S.; Yin, H.; Stephenson, M.; Zong, R.; Chouai, A.; Dorsey, C.; Hennigar, R.; Thummel, R. P.; McFarland, S. A. Exploitation of Long-Lived ${ }^{3}$ IL 
Excited States for Metal-Organic Photodynamic Therapy: Verification in a Metastatic Melanoma Model. J. Am. Chem. Soc. 2013, 135, 17161-17175.

(32) Wang, Z.; Ivanov, M.; Gao, Y.; Bussotti, L.; Foggi, P.; Zhang, H.; Russo, N.; Dick, B.; Zhao, J.; Di Donato, M.; Mazzone, G.; Luo, L.; Fedin, M. Spin-Orbit Charge-Transfer Intersystem Crossing (ISC) in Compact Electron Donor-Acceptor Dyads: ISC Mechanism and Application as Novel and Potent Photodynamic Therapy Reagents. Chem.-Eur. J. 2020, 26, $1091-1102$.

(33) Wang, P.; Guo, S.; Wang, H.-J.; Chen, K.-K.; Zhang, N.; Zhang, Z.-M.; Lu, T.-B. A Broadband and Strong Visible-Light-Absorbing Photosensitizer Boosts Hydrogen Evolution. Nat. Commun. 2019, 10, 3155.

(34) Yuan, Y.-J.; Zhang, J.-Y.; Yu, Z.-T.; Feng, J.-Y.; Luo, W.-J.; Ye, J.-H.; Zou, Z.-G. Impact of Ligand Modification on Hydrogen Photogeneration and Light-Harvesting Applications Using Cyclometalated Iridium Complexes. Inorg. Chem. 2012, 51, 4123-4133.

(35) Ji, S.; Wu, W.; Wu, W.; Guo, H.; Zhao, J. Ruthenium(II) Polyimine Complexes with a Long-Lived ${ }^{3} \mathrm{IL}$ Excited State or a ${ }^{3} \mathrm{MLCT} /{ }^{3} \mathrm{IL}$ Equilibrium: Efficient Triplet Sensitizers for Low-Power Upconversion. Angew. Chem., Int. Ed. 2011, 50, 1626-1629.

(36) Ventura, B.; Marconi, G.; Bröring, M.; Krüger, R.; Flamigni, L. Bis(BF $\left(\mathrm{BF}_{2}\right)-2,2^{\text {' }}$ Bidipyrrins, a Class of Bodipy Dyes with New Spectroscopic and Photophysical Properties. New J. Chem. 2009, 33, 428-438. 
(37) Bröring, M.; Krüger, R.; Link, S.; Kleeberg, C.; Köhler, S.; Xie, X.; Ventura, B.; Flamigni, L. Bis(BF $)$-2,2'-Bidipyrrins (BisBODIPYs): Highly Fluorescent Bodipy Dimers with Large Stokes Shifts. Chem. -Eur. J. 2008, 14, 2976-2983.

(38) Wu, W.; Zhao, J.; Sun, J.; Guo, S. Light-Harvesting Fullerene Dyads as Organic Triplet Photosensitizers for Triplet-Triplet Annihilation Upconversions. J. Org. Chem. 2012, 77, $5305-5312$.

(39) Huang, L.; Cui, X.; Therrien, B.; Zhao, J. Energy-Funneling-Based Broadband VisibleLight-Absorbing Bodipy- $\mathrm{C}_{60}$ Triads and Tetrads as Dual Functional Heavy-Atom-Free Organic Triplet Photosensitizers for Photocatalytic Organic Reactions. Chem. -Eur. J. 2013, $19,17472-17482$.

(40) Filatov, M. A.; Karuthedath, S.; Polestshuk, P. M.; Savoie, H.; Flanagan, K. J.; Sy, C.; Sitte, E.; Telitchko, M.; Laquai, F.; Boyle, R. W.; Senge, M. O. Generation of Triplet Excited States Via Photoinduced Electron Transfer in Meso-Anthra-Bodipy: Fluorogenic Response toward Singlet Oxygen in Solution and in Vitro. J. Am. Chem. Soc. 2017, 139, 6282-6285.

(41) Wang, Z.; Zhao, J. Bodipy-Anthracene Dyads as Triplet Photosensitizers: Effect of Chromophore Orientation on Triplet-State Formation Efficiency and Application in TripletTriplet Annihilation Upconversion. Org. Lett. 2017, 19, 4492-4495.

(42) Hu, W.; Liu, M.; Zhang, X.-F.; Wang, Y.; Wang, Y.; Lan, H.; Zhao, H. Can BodipyElectron Acceptor Conjugates Act as Heavy Atom-Free Excited Triplet State and Singlet 
Oxygen Photosensitizers Via Photoinduced Charge Separation-Charge Recombination Mechanism? J. Phys. Chem. C 2019, 123, 15944-15955.

(43) Wang, Z.; Sukhanov, A. A.; Toffoletti, A.; Sadiq, F.; Zhao, J.; Barbon, A.; Voronkova, V. K.; Dick, B. Insights into the Efficient Intersystem Crossing of Bodipy-Anthracene Compact Dyads with Steady-State and Time-Resolved Optical/Magnetic Spectroscopies and Observation of the Delayed Fluorescence. J. Phys. Chem. C 2019, 123, 265-274.

(44) Smith, M. B.; Michl, J. Singlet Fission. Chem. Rev. 2010, 110, 6891-6936.

(45) Nagarajan, K.; Mallia, A. R.; Reddy, V. S.; Hariharan, M. Access to Triplet Excited State in Core-Twisted Perylenediimide. J. Phys. Chem. C 2016, 120, 8443-8450.

(46) Liu, H.; Wang, R.; Shen, L.; Xu, Y.; Xiao, M.; Zhang, C.; Li, X. A Covalently Linked Tetracene Trimer: Synthesis and Singlet Exciton Fission Property. Org. Lett. 2017, 19, $580-583$.

(47) Yu, Z.; Wu, Y.; Peng, Q.; Sun, C.; Chen, J.; Yao, J.; Fu, H. Accessing the Triplet State in Heavy-Atom-Free Perylene Diimides. Chem. Eur. J. 2016, 22, 4717-4722.

(48) Yang, W.; Zhao, J.; Sonn, C.; Escudero, D.; Karatay, A.; Yaglioglu, H. G.; Küçüköz, B.; Hayvali, M.; Li, C.; Jacquemin, D. Efficient Intersystem Crossing in Heavy-Atom-Free Perylenebisimide Derivatives. J. Phys. Chem. C 2016, 120, 10162-10175.

(49) Yushchenko, O.; Licari, G.; Mosquera-Vazquez, S.; Sakai, N.; Matile, S.; Vauthey, E. Ultrafast Intersystem-Crossing Dynamics and Breakdown of the Kasha-Vavilov's Rule of Naphthalenediimides. J. Phys. Chem. Lett. 2015, 6, 2096-2100. 
(50) Wang, Z.; Zhao, J.; Barbon, A.; Toffoletti, A.; Liu, Y.; An, Y.; Xu, L.; Karatay, A.; Yaglioglu, H. G.; Yildiz, E. A.; Hayvali, M. Radical-Enhanced Intersystem Crossing in New Bodipy Derivatives and Application for Efficient Triplet-Triplet Annihilation Upconversion. J. Am. Chem. Soc. 2017, 139, 7831-7842.

(51) Hussain, M.; Taddei, M.; Bussotti, L.; Foggi, P.; Zhao, J.; Liu, Q.; Di Donato, M. Intersystem Crossing in Naphthalenediimide-Oxoverdazyl Dyads: Synthesis and Study of the Photophysical Properties. Chem. -Eur. J. 2019, 25, 15615-15627.

(52) Sapir, M.; Donckt, E. V. Intersystem Crossing in the Helicenes. Chem. Phys. Lett. 1975, $36,108-110$.

(53) Schmidt, K.; Brovelli, S.; Coropceanu, V.; Beljonne, D.; Cornil, J.; Bazzini, C.; Caronna, T.; Tubino, R.; Meinardi, F.; Shuai, Z.; Brédas, J.-L. Intersystem Crossing Processes in Nonplanar Aromatic Heterocyclic Molecules. J. Phys. Chem. A 2007, 111, 10490-10499.

(54) Wu, Y.; Zhen, Y.; Ma, Y.; Zheng, R.; Wang, Z.; Fu, H. Exceptional Intersystem Crossing in Di(perylene bisimide)s: A Structural Platform toward Photosensitizers for Singlet Oxygen Generation. J. Phys. Chem. Lett. 2010, 1, 2499-2502.

(55) Nagarajan, K.; Mallia, A. R.; Muraleedharan, K.; Hariharan, M. Enhanced Intersystem Crossing in Core-Twisted Aromatics. Chem. Sci. 2017, 8, 1776-1782.

(56) Lim, J. M.; Yoon, Z. S.; Shin, J.-Y.; Kim, K. S.; Yoon, M.-C.; Kim, D. The Photophysical Properties of Expanded Porphyrins: Relationships between Aromaticity, Molecular Geometry and Non-Linear Optical Properties. Chem. Commun. 2008, 261-273. 
(57) Dong, Y.; Dick, B.; Zhao, J. Twisted Bodipy Derivative as a Heavy-Atom-Free Triplet Photosensitizer Showing Strong Absorption of Yellow Light, Intersystem Crossing, and a High-Energy Long-Lived Triplet State. Org. Lett. 2020, 22, 5535-5539.

(58) Wang, Z.; Huang, L.; Yan, Y.; El-Zohry, A. M.; Toffoletti, A.; Zhao, J.; Barbon, A.; Dick, B.; Mohammed, O. F.; Han, G. Elucidation of the Intersystem Crossing Mechanism in a Helical Bodipy for Low-Dose Photodynamic Therapy. Angew. Chem., Int. Ed. 2020, 59, $16114-16121$.

(59) Ito, H.; Sakai, H.; Suzuki, Y.; Kawamata, J.; Hasobe, T. Systematic Control of Structural and Photophysical Properties of $\pi$-Extended Mono- and Bis-Bodipy Derivatives. Chem. -Eur. J. 2020, 26, 316-325.

(60)Loudet, A.; Burgess, K. Bodipy Dyes and Their Derivatives: Syntheses and Spectroscopic Properties. Chem. Rev. 2007, 107, 4891-4932.

(61) Ulrich, G.; Ziessel, R.; Harriman, A. The Chemistry of Fluorescent Bodipy Dyes: Versatility Unsurpassed. Angew. Chem., Int. Ed. 2008, 47, 1184-1201.

(62) Lu, H.; Mack, J.; Yang, Y.; Shen, Z. Structural Modification Strategies for the Rational Design of Red/NIR Region Bodipys. Chem. Soc. Rev. 2014, 43, 4778-4823.

(63) Lakshmi, V.; Rajeswara Rao, M.; Ravikanth, M. Halogenated Boron-Dipyrromethenes: Synthesis, Properties and Applications. Org. Biomol. Chem. 2015, 13, 2501-2517.

(64) Razi, S. S.; Koo, Y. H.; Kim, W.; Yang, W.; Wang, Z.; Gobeze, H.; D’Souza, F.; Zhao, J.; Kim, D. Ping-Pong Energy Transfer in a Boron Dipyrromethane Containing Pt(II)-Schiff 
Base Complex: Synthesis, Photophysical Studies, and Anti-Stokes Shift Increase in TripletTriplet Annihilation Upconversion. Inorg. Chem. 2018, 57, 4877-4890.

(65) Filatov, M. A.; Karuthedath, S.; Polestshuk, P. M.; Callaghan, S.; Flanagan, K. J.; Wiesner, T.; Laquai, F.; Senge, M. O. Bodipy-Pyrene and Perylene Dyads as Heavy-AtomFree Singlet Oxygen Sensitizers. ChemPhotoChem 2018, 2, 606-615.

(66) Zhang, X.-F.; Feng, N. Photoinduced Electron Transfer-Based Halogen-Free Photosensitizers: Covalent Meso-Aryl (Phenyl, Naphthyl, Anthryl, and Pyrenyl) as Electron Donors to Effectively Induce the Formation of the Excited Triplet State and Singlet Oxygen for Bodipy Compounds. Chem. -Asian J. 2017, 12, 2447-2456.

(67) Hou, Y.; Liu, Q.; Zhao, J. An Exceptionally Long-Lived Triplet State of Red LightAbsorbing Compact Phenothiazine-Styrylbodipy Electron Donor/Acceptor Dyads: A Better Alternative to the Heavy Atom-Effect? Chem. Commun. 2020, 56, 1721-1724.

(68) Ni, Y.; Zeng, W.; Huang, K.-W.; Wu, J. Benzene-Fused Bodipys: Synthesis and the Impact of Fusion Mode. Chem. Commun. 2013, 49, 1217-1219.

(69) Zhao, N.; Xuan, S.; Zhou, Z.; Fronczek, F. R.; Smith, K. M.; Vicente, M. G. H. Synthesis and Spectroscopic and Cellular Properties of near-IR $[a]$ Phenanthrene-Fused 4,4Difluoro-4-Bora-3a,4a-Diaza-S-Indacenes. J. Org. Chem. 2017, 82, 9744-9750.

(70) Yogo, T.; Urano, Y.; Ishitsuka, Y.; Maniwa, F.; Nagano, T. Highly Efficient and Photostable Photosensitizer Based on Bodipy Chromophore. J. Am. Chem. Soc. 2005, 127, $12162-12163$. 
(71) Ziessel, R.; Harriman, A. Artificial Light-Harvesting Antennae: Electronic Energy Transfer by Way of Molecular Funnels. Chem. Commun. 2011, 47, 611-631.

(72) Cakmak, Y.; Kolemen, S.; Duman, S.; Dede, Y.; Dolen, Y.; Kilic, B.; Kostereli, Z.; Yildirim, L. T.; Dogan, A. L.; Guc, D.; Akkaya, E. U. Designing Excited States: TheoryGuided Access to Efficient Photosensitizers for Photodynamic Action. Angew. Chem., Int. Ed. 2011, 50, 11937-11941.

(73) Rao, M. R.; Mobin, S. M.; Ravikanth, M. Boron-Dipyrromethene Based Specific Chemodosimeter for Fluoride Ion. Tetrahedron 2010, 66, 1728-1734.

(74) Lou, Z.; Hou, Y.; Chen, K.; Zhao, J.; Ji, S.; Zhong, F.; Dede, Y.; Dick, B. Different Quenching Effect of Intramolecular Rotation on the Singlet and Triplet Excited States of Bodipy. J. Phys. Chem. C 2018, 122, 185-193.

(75) Li, X.; Gong, C.; Gurzadyan, G. G.; Gelin, M. F.; Liu, J.; Sun, L. Ultrafast Relaxation Dynamics in Zinc Tetraphenylporphyrin Surface-Mounted Metal Organic Framework. J. Phys. Chem. C 2018, 122, 50-61.

(76) Sheldrick, G. M. SADABS, “Empirical Absorption Correction Program,” University of Göttingen, Göttingen, 1996.

(77) Sheldrick, G. M. A Short History of SHELX. Acta Crystallogr, 2008, 64, 112-122.

(78) Sheldrick, G. M. SHELXS 97, Program for Crystal Structure Refinement; University of Gottingen: Gottingen, Germany, 1997. 
(79) TURBOMOLE V6.6 2014, a development of University of Karlsruhe and Forschungszentrum Karlsruhe GmbH, 1989-2007, TURBOMOLE GmbH, since 2007; available from http://www.turbomole.com.

(80) Rinkevicius, Z.; Tunell, I.; Sałek, P.; Vahtras, O.; Ågren, H. Restricted Density Functional Theory of Linear Time-Dependent Properties in Open-Shell Molecules. J. Chem. Phys. 2003, 119, 34-46.

(81) Ågren, H.; Vahtras, O.; Minaev, B. Response Theory and Calculations of Spin-Orbit Coupling Phenomena in Molecules. In Adv. Quantum. Chem., Löwdin, P.-O.; Sabin, J. R.; Zerner, M. C. Eds. Academic Press: 1996, 27, 71-162.

(82) Helgaker, T. J., P.; Olsen, J.; Ruud, K.; Andersen, T.; Bak, K. L.; Bakken, V.; Christiansen, O.; Dahle, P.; Dalskov, E. K.; Enevoldsen, T.; Heiberg, H.; Hettema, H.; Jonsson, D.; Kirpekar, S.; Kobayashi, R.; Koch, H.; Mikkelsen, K. V.; Norman, P.; Packer, M. J.; Saue, T.; Taylor, P. R.; Vahtras, O.; Jensen, H. J. A.; Ågren, H. Dalton: An Ab Initio Electronic Structure Program, version 1.0, 1997.

(83) Stoll, S.; Schweiger, A. a Comprehensive Software Package for Spectral Simulation and Analysis in EPR. J. Magn. Reson. 2006, 178, 42-55.

(84) Ceola, S.; Franco, L.; Maggini, M.; Corvaja, C. Optical Spectrum of C ${ }_{60}$ Mono-Adducts: Assignment of Transition Bands Using Time-Resolved EPR Magneto-Photo-Selection. Photochem. Photobiol. Sci. 2006, 5, 1177-1182. 
(85) Frath, D.; Massue, J.; Ulrich, G.; Ziessel, R. Luminescent Materials: Locking $\pi-$ Conjugated and Heterocyclic Ligands with Boron(III). Angew. Chem., Int. Ed. 2014, 53, $2290-2310$.

(86) Zhou, Z.; Zhou, J.; Gai, L.; Yuan, A.; Shen, Z. Naphtho[b]-Fused Bodipys: One Pot Suzuki-Miyaura-Knoevenagel Synthesis and Photophysical Properties. Chem. Commun. 2017, $53,6621-6624$.

(87) Kee, H. L.; Kirmaier, C.; Yu, L.; Thamyongkit, P.; Youngblood, W. J.; Calder, M. E.; Ramos, L.; Noll, B. C.; Bocian, D. F.; Scheidt, W. R.; Birge, R. R.; Lindsey, J. S.; Holten, D. Structural Control of the Photodynamics of Boron-Dipyrrin Complexes. J. Phys. Chem. B 2005, 109, 20433-20443.

(88) Suhina, T.; Amirjalayer, S.; Woutersen, S.; Bonn, D.; Brouwer, A. M. Ultrafast Dynamics and Solvent-Dependent Deactivation Kinetics of Bodipy Molecular Rotors. Phys. Chem. Chem. Phys. 2017, 19, 19998-20007.

(89) Kuimova, M. K.; Yahioglu, G.; Levitt, J. A.; Suhling, K. Molecular Rotor Measures Viscosity of Live Cells Via Fluorescence Lifetime Imaging. J. Am. Chem. Soc. 2008, 130, $6672-6673$.

(90) Escudero, D. Revising Intramolecular Photoinduced Electron Transfer (PET) from First-Principles. Acc. Chem. Res. 2016, 49, 1816-1824.

(91) DeRosa, M. C.; Crutchley, R. J. Photosensitized Singlet Oxygen and Its Applications. Coord. Chem. Rev. 2002, 233-234, 351-371. 
(92) Li, Y.; Dandu, N.; Liu, R.; Li, Z.; Kilina, S.; Sun, W. Effects of Extended $\pi$ Conjugation in Phenanthroline $\left(\mathrm{N}^{\wedge} \mathrm{N}\right)$ and Phenylpyridine $\left(\mathrm{C}^{\wedge} \mathrm{N}\right)$ Ligands on the Photophysics and Reverse Saturable Absorption of Cationic Heteroleptic Iridium(III) Complexes. J. Phys. Chem. C 2014, 118, 6372-6384.

(93) van Stokkum, I. H. M.; Larsen, D. S.; van Grondelle, R. Global and Target Analysis of Time-Resolved Spectra. Biochimica et Biophysica Acta (BBA) - Bioenergetics 2004, 1657, $82-104$.

(94) Prieto-Montero, R.; Sola-Llano, R.; Montero, R.; Longarte, A.; Arbeloa, T.; LópezArbeloa, I.; Martínez-Martínez, V.; Lacombe, S. Methylthio Bodipy as a Standard Triplet Photosensitizer for Singlet Oxygen Production: A Photophysical Study. Phys. Chem. Chem. Phys. 2019, 21, 20403-20414.

(95) Sabatini, R. P.; McCormick, T. M.; Lazarides, T.; Wilson, K. C.; Eisenberg, R.; McCamant, D. W. Intersystem Crossing in Halogenated Bodipy Chromophores Used for Solar Hydrogen Production. J. Phys. Chem. Lett. 2011, 2, 223-227.

(96) M. Montalti; A. Credi; L. Prodi; Gandolfi, M. T. Handbook of Photochemistry, (CRC press, Boca Raton), 2006.

(97) Zhang, T.; Ma, X.; Tian, H. A Facile Way to Obtain near-Infrared Room-Temperature Phosphorescent Soft Materials Based on Bodipy Dyes. Chem. Sci. 2020, 11, 482-487. 
(98) Wu, W.; Guo, H.; Wu, W.; Ji, S.; Zhao, J. Organic Triplet Sensitizer Library Derived from a Single Chromophore (Bodipy) with Long-Lived Triplet Excited State for TripletTriplet Annihilation Based Upconversion. J. Org. Chem. 2011, 76, 7056-7064.

(99) Mahmood, Z.; Zhao, J. The Unquenched Triplet Excited State of the Fluorescent OFF/ON Bodipy-Derived Molecular Probe Based on Photo-Induced Electron Transfer. Photochem. Photobiol. Sci. 2016, 15, 1358-1365.

(100) Zhang, X.-F.; Yang, X.; Niu, K.; Geng, H. Phosphorescence of Bodipy Dyes. J. Photochem. Photobiol. A 2014, 285, 16-20.

(101) Wang, Z.; Zhao, J.; Di Donato, M.; Mazzone, G. Increasing the Anti-Stokes Shift in TTA Upconversion with Photosensitizers Showing Red-Shifted Spin-Allowed Charge Transfer Absorption but a Non-Compromised Triplet State Energy Level. Chem. Commun. 2019, 55, $1510-1513$.

(102) Kim, J.-H.; Deng, F.; Castellano, F. N.; Kim, J.-H. High Efficiency Low-Power Upconverting Soft Materials. Chem. Mater. 2012, 24, 2250-2252.

(103) Richert, S.; Tait, C. E.; Timmel, C. R. Delocalisation of Photoexcited Triplet States Probed by Transient EPR and Hyperfine Spectroscopy. J. Magn. Reson. 2017, 280, 103-116. (104) Kawai, A.; Shibuya, K. Electron Spin Dynamics in a Pair Interaction between Radical and Electronically-Excited Molecule as Studied by a Time-Resolved ESR Method. J. Photochem. Photobiol., C 2006, 7, 89-103.

(105) Weber, S. Transient EPR. In eMagRes, 2017, 6, 255-270. 
(106) El-Sayed, M. A.; Siegel, S. Method of "Magnetophotoselection" of the Lowest Excited Triplet State of Aromatic Molecules. J. Chem. Phys. 1966, 44, 1416-1423.

(107) Toffoletti, A.; Wang, Z.; Zhao, J.; Tommasini, M.; Barbon, A. Precise Determination of the Orientation of the Transition Dipole Moment in a Bodipy Derivative by Analysis of the Magnetophotoselection Effect. Phys. Chem. Chem. Phys. 2018, 20, 20497-20503.

(108) Hou, Y.; Kurganskii, I.; Elmali, A.; Zhang, H.; Gao, Y.; Lv, L.; Zhao, J.; Karatay, A.; Luo, L.; Fedin, M. Electronic Coupling and Spin-Orbit Charge Transfer Intersystem Crossing (SOCT-ISC) in Compact BDP-Carbazole Dyads with Different Mutual Orientations of the Electron Donor and Acceptor. J. Chem. Phys. 2020, 152, 114701.

(109) Hintze, C.; Korf, P.; Degen, F.; Schütze, F.; Mecking, S.; Steiner, U. E.; Drescher, M. Delocalization of Coherent Triplet Excitons in Linear Rigid Rod Conjugated Oligomers. $J$. Phys. Chem. Lett. 2017, 8, 690-695.

(110) Tanabe, M.; Matsuoka, H.; Ohba, Y.; Yamauchi, S.; Sugisaki, K.; Toyota, K.; Sato, K.; Takui, T.; Goldberg, I.; Saltsman, I.; Gross, Z. Time-Resolved Electron Paramagnetic Resonance and Phosphorescence Studies of the Lowest Excited Triplet States of Rh(III) Corrole Complexes. J. Phys. Chem. A 2012, 116, 9662-9673.

(111) Dance, Z. E. X.; Mickley, S. M.; Wilson, T. M.; Ricks, A. B.; Scott, A. M.; Ratner, M. A.; Wasielewski, M. R. Intersystem Crossing Mediated by Photoinduced Intramolecular Charge Transfer: Julolidine-Anthracene Molecules with Perpendicular $\pi$ Systems. J. Phys. Chem. A 2008, 112, 4194-4201. 
(112) Hou, Y.; Zhang, X.; Chen, K.; Liu, D.; Wang, Z.; Liu, Q.; Zhao, J.; Barbon, A. Charge Separation, Charge Recombination, Long-Lived Charge Transfer State Formation and Intersystem Crossing in Organic Electron Donor/Acceptor Dyads. J. Mater. Chem. C 2019, 7, $12048-12074$.

(113) Barbon, A.; Dal Farra, M. G.; Ciuti, S.; Albertini, M.; Bolzonello, L.; Orian, L.; Di Valentin, M. Comprehensive Investigation of the Triplet State Electronic Structure of FreeBase 5,10,15,20-Tetrakis(4-Sulfonatophenyl)Porphyrin by a Combined Advanced EPR and Theoretical Approach. J. Chem. Phys. 2020, 152, 034201.

(114) Wang, Y.-W.; Descalzo, A. B.; Shen, Z.; You, X.-Z.; Rurack, K. DihydronaphthaleneFused Boron-Dipyrromethene (Bodipy) Dyes: Insight into the Electronic and Conformational Tuning Modes of Bodipy Fluorophores. Chem. -Eur. J. 2010, 16, 2887-2903.

(115) Tait, C. E.; Neuhaus, P.; Anderson, H. L.; Timmel, C. R. Triplet State Delocalization in a Conjugated Porphyrin Dimer Probed by Transient Electron Paramagnetic Resonance Techniques. J. Am. Chem. Soc. 2015, 137, 6670-6679.

(116) Laurent, A. D.; Jacquemin, D. TD-DFT Benchmarks: A Review. Int. J. Quantum. Chem. 2013, 113, 2019-2039.

(117) Loos, P.-F.; Scemama, A.; Jacquemin, D. The Quest for Highly Accurate Excitation Energies: A Computational Perspective. J. Phys. Chem. Lett. 2020, 11, 2374-2383. 
(118) Dreuw, A.; Wormit, M. The Algebraic Diagrammatic Construction Scheme for the Polarization Propagator for the Calculation of Excited States. WIREs Computational Molecular Science 2015, 5, 82-95.

(119) Chibani, S.; Le Guennic, B.; Charaf-Eddin, A.; Laurent, A. D.; Jacquemin, D. Revisiting the Optical Signatures of Bodipy with ab initio Tools. Chem. Sci. 2013, 4, 1950-963. (120) Veys, K.; Escudero, D. Computational Protocol to Predict Anti-Kasha Emissions: The Case of Azulene Derivatives. J. Phys. Chem. A 2020, 124, 7228-7237.

\section{Table of Content:}

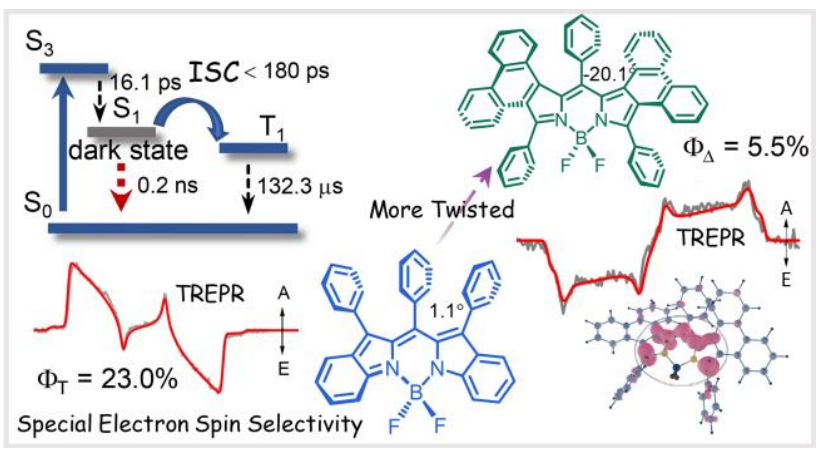

